\title{
Maternal complications following open and fetoscopic fetal surgery: A systematic review and meta-analysis
}

\author{
Adalina Sacco ${ }^{1}$ (D) I Lennart Van der Veeken ${ }^{2}$ | Emma Bagshaw ${ }^{1}$ | Catherine Ferguson ${ }^{1}$ । \\ Tim Van Mieghem ${ }^{3}$ (D) । Anna L. David ${ }^{1,2,4}$ (D) । Jan Deprest ${ }^{1,2,5}$
}

\author{
${ }^{1}$ Department of Maternal and Fetal Medicine, \\ Institute for Women's Health, University \\ College London, London, UK \\ ${ }^{2}$ Department of Development and \\ Regeneration, Cluster Woman and Child, \\ Biomedical Sciences, KU Leuven, Leuven, \\ Belgium \\ ${ }^{3}$ Department of Obstetrics and Gynaecology, \\ Mount Sinai Hospital and University of \\ Toronto, Toronto, Ontario, Canada \\ ${ }^{4}$ National Institute for Health Research, \\ University College London Hospitals \\ Biomedical Research Centre, London, UK \\ ${ }^{5}$ Clinical Department Obstetrics and \\ Gynaecology, University Hospitals Leuven, \\ Leuven, Belgium

\section{Correspondence} \\ Adalina Sacco, Fetal Medicine Unit, Elizabeth \\ Garrett Anderson Wing, University College \\ London Hospital, 235 Euston Road, London \\ NW1 2BU, UK. \\ Email: a.sacco@ucl.ac.uk \\ Funding information \\ Erasmus + Programme of the European Union, \\ Grant/Award Number: 2013-0040; Engineer- \\ ing and Physical Sciences Research Council, \\ Grant/Award Number: NS/A000027/1; \\ Wellcome Trust, Grant/Award Number: \\ WT101957
}

\begin{abstract}
Objective: To establish maternal complication rates for fetoscopic or open fetal surgery.

Methods: We conducted a systematic literature review for studies of fetoscopic or open fetal surgery performed since 1990, recording maternal complications during fetal surgery, the remainder of pregnancy, delivery, and after the index pregnancy.

Results: One hundred sixty-six studies were included, reporting outcomes for open fetal ( $n=1193$ patients) and fetoscopic surgery ( $n=9403$ patients). No maternal deaths were reported. The risk of any maternal complication in the index pregnancy was $20.9 \%(95 \% \mathrm{Cl}, 15.22-27.13)$ for open fetal and $6.2 \%(95 \% \mathrm{Cl}, 4.93-7.49)$ for fetoscopic surgery. For severe maternal complications (grades III to V Clavien-Dindo classification of surgical complications), the risk was 4.5\% (95\% Cl 3.24-5.98) for open fetal and $1.7 \%$ (95\% Cl, 1.19-2.20) for fetoscopic surgery. In subsequent pregnancies, open fetal surgery increased the risk of preterm birth but not uterine dehiscence or rupture. Nearly one quarter of reviewed studies ( $n=175,23.3 \%$ ) was excluded for failing to report the presence or absence of maternal complications.

Conclusions: Maternal complications occur in 6.2\% fetoscopic and $20.9 \%$ open fetal surgeries, with serious maternal complications in $1.7 \%$ fetoscopic and $4.5 \%$ open procedures.

Reporting of maternal complications is variable. To properly quantify maternal risks, outcomes should be reported consistently across all fetal surgery studies.
\end{abstract}

\section{1 | INTRODUCTION}

The last 35 years have witnessed an expansion of fetal therapy options, ${ }^{1,2}$ with surgery on the fetus, placenta, or cord now relatively common in tertiary-level fetal medicine units. Enabled by advancements in imaging, surgical instrumentation and techniques, early diagnosis, and treatment of fetal anomalies are now possible for a wide range of conditions. ${ }^{3}$

The mother has been called an "innocent bystander" in fetal surgery, ${ }^{4}$ and generally, fetal therapy is almost exclusively offered to women who are healthy themselves. Fetal surgery poses risks to the mother not only during the procedure itself but also throughout the

This is an open access article under the terms of the Creative Commons Attribution License, which permits use, distribution and reproduction in any medium, provided the original work is properly cited.

(c) 2019 The Authors. Prenatal Diagnosis Published by John Wiley \& Sons Ltd. 
remainder of the index pregnancy, potentially during any future pregnancies and throughout the woman's entire life. Fetal surgery offers no direct medical benefit to the mother, and from an ethical perspective, maternal risks should be minor and acceptable to the mother and family. ${ }^{5}$

Information regarding safety of surgery is important for counselling and informed decision making; however, robust data on maternal complications of fetal surgery are lacking. One single-centre study of maternal outcomes following both open fetal and fetoscopic surgery performed between 1989 and 2003 found a number of short-term morbidities. ${ }^{6}$ A systematic review of maternal complications following fetoscopic laser coagulation for twin-to-twin transfusion syndrome (TTTS) in 1785 patients treated between ${ }^{7} 1990$ and 2009 observed an overall adverse event rate of $5.4 \%$ with severe complications in $1.0 \%$. The aim of this study was to estimate the incidence of immediate and long-term maternal complications of fetoscopic or open fetal surgery through a systematic review of the literature.

\section{2 | METHODS}

\section{1 | Protocol and registration}

This systematic review was conducted in accordance with preferred reporting items for systematic reviews and meta-analyses (PRISMA) guidance. ${ }^{8}$ The protocol was registered with the international prospective register of systematic reviews (PROSPEROCRD42017082411).

\section{2 | Eligibility criteria}

All randomised, cohort, and case-controlled studies and case series reporting the results of open fetal or fetoscopic fetal surgery in humans from January 1990 to October 2018 were considered eligible. No language restrictions were applied. Systematic reviews, narrative review articles, and case reports were excluded. There is no accepted numerical definition of a case series. ${ }^{9}$ We used an empirical cut-off of at least three cases because of the rarity of some procedures and conditions searched for.

\section{3 | Search strategy}

A systematic review was conducted in MEDLINE, EMBASE, and Cochrane databases using free text and Medical Subject Headings (MESH). The electronic search strategy is described in the Supporting Information. Subsequently, a grey literature (first 100 hits in PubMed and Google Scholar) search was performed, and reference lists of relevant review articles were manually checked. Covidence software (Veritas Health Innovation Ltd, Melbourne, Australia) was used to eliminate duplicate articles and manage study screening.

\section{What's already known about this topic?}

- Fetal surgery, both open and fetoscopic, is now widely performed.

- Fetoscopy is perceived as safe for the mother, although specific data on maternal complications is lacking.

- Open fetal surgery is known to cause maternal morbidity, but the exact nature and frequency of complications is not well established across different centres and types of surgery.

\section{What does this study add?}

- This study estimates the nature and frequency of maternal complications following fetoscopic and open fetal surgery.

- For open fetal surgery, the severe complication rate (grades III to V according to the Clavien-Dindo classification of surgical complications) is approximately $4 \%$ and minor complication rate is $16 \%$.

- For fetoscopic fetal surgery, the severe complication rate is approximately $2 \%$ and minor complication rate is $4 \%$.

\section{4 | Study selection}

Two authors (A.S. and L.VdV.) screened all titles and abstracts independently, excluded irrelevant studies and then independently assessed the remaining full-text articles for eligibility; disagreements were resolved by consensus. Studies were excluded if the full text was unavailable online and the abstract contained insufficient information. Studies with interventions which were not fully described or were performed on the neonate instead of the fetus were excluded. Interventions involving access to the uterus using a device with a total outer diameter of less than $1.5 \mathrm{~mm}$ were excluded; this cut-off was chosen to avoid procedures performed with needles only (eg, amniocentesis, fetal blood transfusion, thoracocentesis or vesicocentesis). Studies of shunting were only included if the outer shunt diameter was greater than or equal to $1.5 \mathrm{~mm}$ or the shunt was inserted fetoscopically. Studies which did not report maternal outcomes were excluded. For the purpose of this study, preterm rupture of membranes (PROM), chorionic membrane separation (CMS), preterm labour, preterm delivery, and gestational age at delivery, although relevant, were not considered to be maternal complications. Studies from which data could not be extracted (eg, composite or combined outcomes given) and studies containing patient cohorts which appeared to have been published previously by the same authors were excluded. 


\section{5 | Data extraction}

Two authors independently extracted data (A.S. and E.B. for open fetal surgery studies, A.S. and C.F. for fetoscopic studies) and entered them into a standardised Excel (Microsoft, Washington, USA) form. Disagreements were resolved by consensus. The ex utero intrapartum treatment (EXIT) procedure was classified as open fetal surgery. Study characteristics noted included study design, underlying fetal condition, type of intervention, presence of a control group, gestational age at surgery, and number of patients in each study. Outcomes recorded for the duration of the index pregnancy included intraoperative complications (maternal death, placental abruption, uterine bleeding/haemorrhage, blood transfusion, organ damage, or anaesthetic complications), post-operative complications (classified from the end of surgery until delivery; maternal death, placental abruption, uterine bleeding/haemorrhage, blood transfusion, sepsis, chorioamnionitis, other infections, pulmonary oedema, amniotic fluid embolism and other respiratory, gastro-intestinal, cardiac, or wound problems), complications at delivery of the index pregnancy (uterine dehiscence or rupture or blood transfusion), and the need for additional treatment at any time during the pregnancy. Outcomes noted at any time following the index pregnancy (late outcomes) included fertility (number of further pregnancies, difficulty conceiving, mean time to conception), future pregnancy complications (miscarriage or preterm delivery), complications during future deliveries (uterine dehiscence or rupture or haemorrhage at delivery), gynaecological and psychological symptoms. When a study reported "haemorrhage" or an actual blood loss of greater than $1000 \mathrm{~mL}$ we noted this as "haemorrhage." This cut-off is an accepted definition of severe bleeding both in pregnancy ${ }^{10}$ and post-partum. ${ }^{11}$ If a study did not specify whether a complication occurred intraoperatively or post-operatively (eg, placental abruption and requirement for blood transfusion), then this was assumed to have occurred post-operatively.

All complications were independently graded according to the Clavien-Dindo classification of surgical complications ${ }^{12}$ by two authors (A.S. and L.VdV.) (Supporting Information). Clavien-Dindo grade I or II complications were defined as mild; grades III to V complications were defined as severe. ${ }^{12}$

\subsection{Quality assessment of studies}

Study quality and risk of bias were analysed by two authors (A.S. and L.VdV.) independently using a standardised form. Randomised trials were analysed using the Cochrane Collaboration's tool for assessing risk of bias. ${ }^{13}$ Case-control studies were analysed using the Newcastle-Ottawa scale for assessing the quality of nonrandomised studies. ${ }^{14}$ Case series were analysed using the National Institutes of Health study quality assessment tool. ${ }^{15}$

\section{7 | Assessment of heterogeneity}

Methodological and clinical heterogeneity of data per study were evaluated. Variables were tested for statistical heterogeneity by applying the $\mathrm{I}^{2}$ test to determine whether data could be pooled. An $\mathrm{I}^{2}$ value less than $40 \%$ was taken to indicate minor heterogeneity; $40 \%$ to $75 \%$ moderate heterogeneity and greater than $75 \%$ substantial heterogeneity. ${ }^{13}$

\section{8 | Meta-analysis}

Meta-analysis for all outcomes was carried out using MedCalc statistical software version 15.4 (MedCalc Software, Ostend, Belgium). Results were expressed as proportions with $95 \%$ confidence intervals (Cl) as all outcomes were categorical variables. Pooled proportions were calculated using both the fixed and random effects model in case of homogeneity or heterogeneity respectively.

\section{3 | RESULTS}

\section{1 | Study selection}

The electronic literature search identified 70367 studies published between 1990 and 2018 (Figure 1); search of the grey literature and reference lists identified a further 16 studies. Following this, 48248 studies were immediately removed as duplicates. The remaining studies (22 135) were screened by title and abstract, and a further 21384 were excluded as irrelevant. Full texts of the remaining 751 articles were reviewed, and 585 were excluded for the following reasons: no reporting of maternal outcomes (175/585, $29.9 \%$ of studies excluded and $23.3 \%$ [175/751] of all studies assessed), insufficient information available (conference abstract/poster only or full text unavailable) $(119 / 585,20.3 \%)$, study design other than randomised trial, casecontrol trial or case series (110/585, 18.8\%), and uterine access using a device less than $1.5 \mathrm{~mm}(59 / 585,10.1 \%)$. Thirty studies were translated from French (10), Spanish (seven), Polish (five), German (three), Dutch (two), Portuguese (two), and Turkish (one), of which 16 were included following review. Two Chinese-language papers were identified, but the full text could not be accessed online. Eventually, 166 studies were included; 41 on open fetal surgery, 122 on fetoscopic surgery, and three studies including both surgery types.

\section{2 | Study characteristics}

Characteristics of included studies are shown in Tables 1-3. Studies of open fetal (Table 1) and fetoscopic (Table 2) surgery are presented and analysed separately as the difference in surgical technique was considered too great for combined analysis. Seven studies specifically focused on late complications, ie, after the index pregnancy and are presented separately (Table 3). The majority of studies $(68.1 \%, 113 / 166)$ were case series, ie, without a control group; $27.1 \%(45 / 166)$ were case control studies, and $4.8 \%$ (8/166) were randomised trials. 


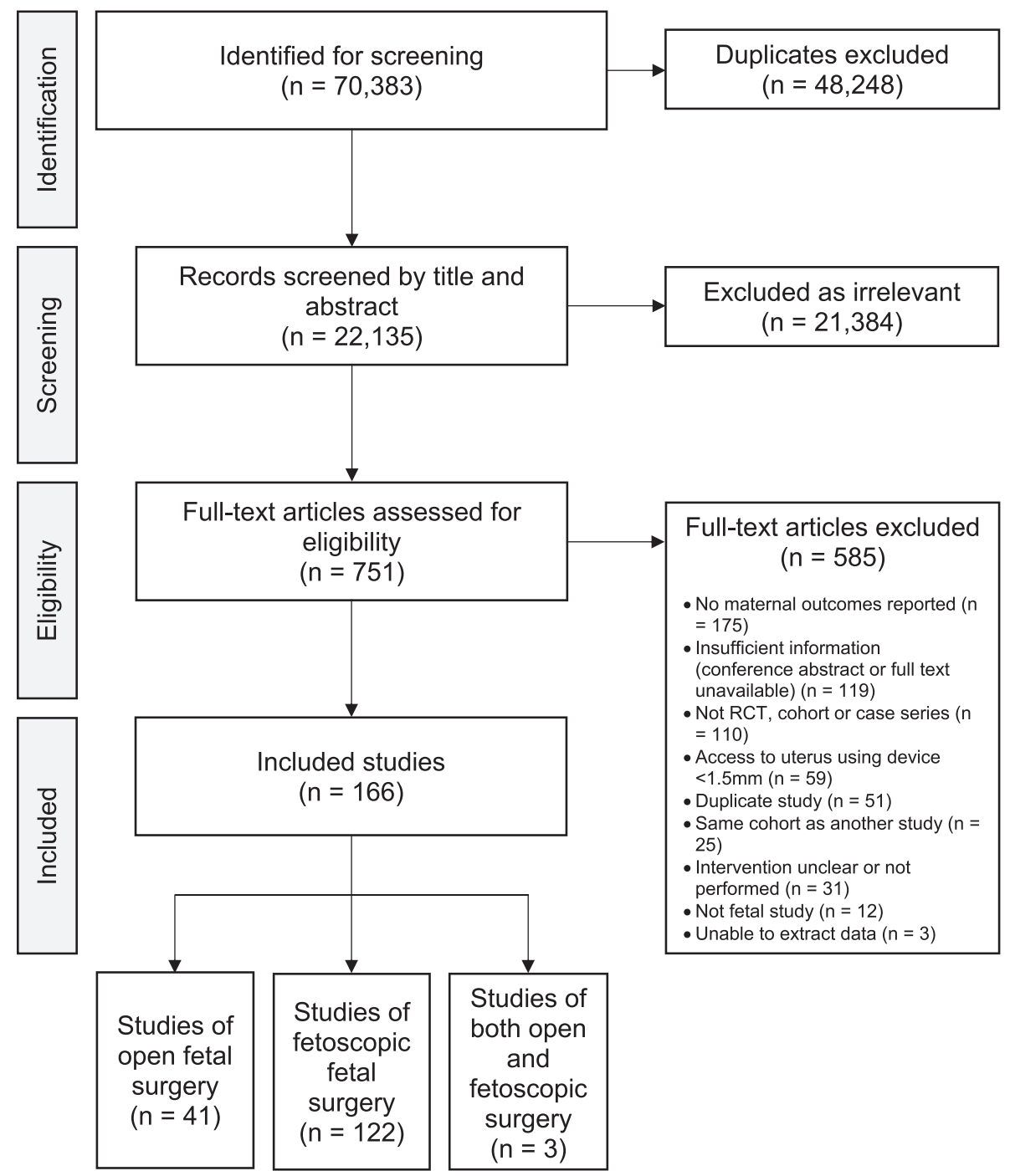

FIGURE 1 Flow diagram of study selection adapted from preferred reporting items for systematic reviews and meta-analyses (PRISMA) ${ }^{8} 2009$

\section{3 | Risk of bias}

Quality assessment of the studies is given in the Supporting Information. Most studies (139/166, 83.7\%) had a low risk of bias or were high quality. All remaining studies $(27 / 166,16.3 \%)$ had an unclear risk of bias or were fair quality. No studies were found to have a high risk of bias or be low quality overall. For randomised trials, included studies had a high risk of bias with regards to blinding. For case control studies, included studies did not describe statistical methods well overall.

\section{4 | Statistical heterogeneity}

Maternal outcome data were pooled in 64 separate meta-analyses, of which $37.5 \%$ (24/64) had no or minor heterogeneity. In 39.1\% (25/ 64), there was moderate heterogeneity, and in $23.4 \%$ (15/64), there was considerable heterogeneity. The levels of heterogeneity per outcome measure are listed in the Supporting Information. As both clinical and statistical heterogeneity were found, pooled proportions were given using the random effects model for meta-analysis.

\section{5 | Maternal complications in the index pregnancy -intraoperative}

Table 4 summarises maternal complications according to type of surgery performed. No maternal deaths (Clavien-Dindo grade $\mathrm{V}$ ) due to fetal surgery were reported in any study (10 596 procedures). One study $^{86}$ reported a patient at 20 weeks' gestation experiencing a cardiorespiratory arrest prior to fetoscopy for laser photocoagulation. The cause was considered to be a combination of morbid obesity, spinal anaesthesia, and aortocaval compression and not related to the procedure, which had not commenced. An immediate delivery was conducted by hysterotomy as part of maternal resuscitation, and the patient made a full recovery. Another study ${ }^{47}$ reported brief maternal seizure-like activity during open fetal surgery, which was thought to be anaesthesia-related. 
TABLE 1 Included studies of open fetal surgery

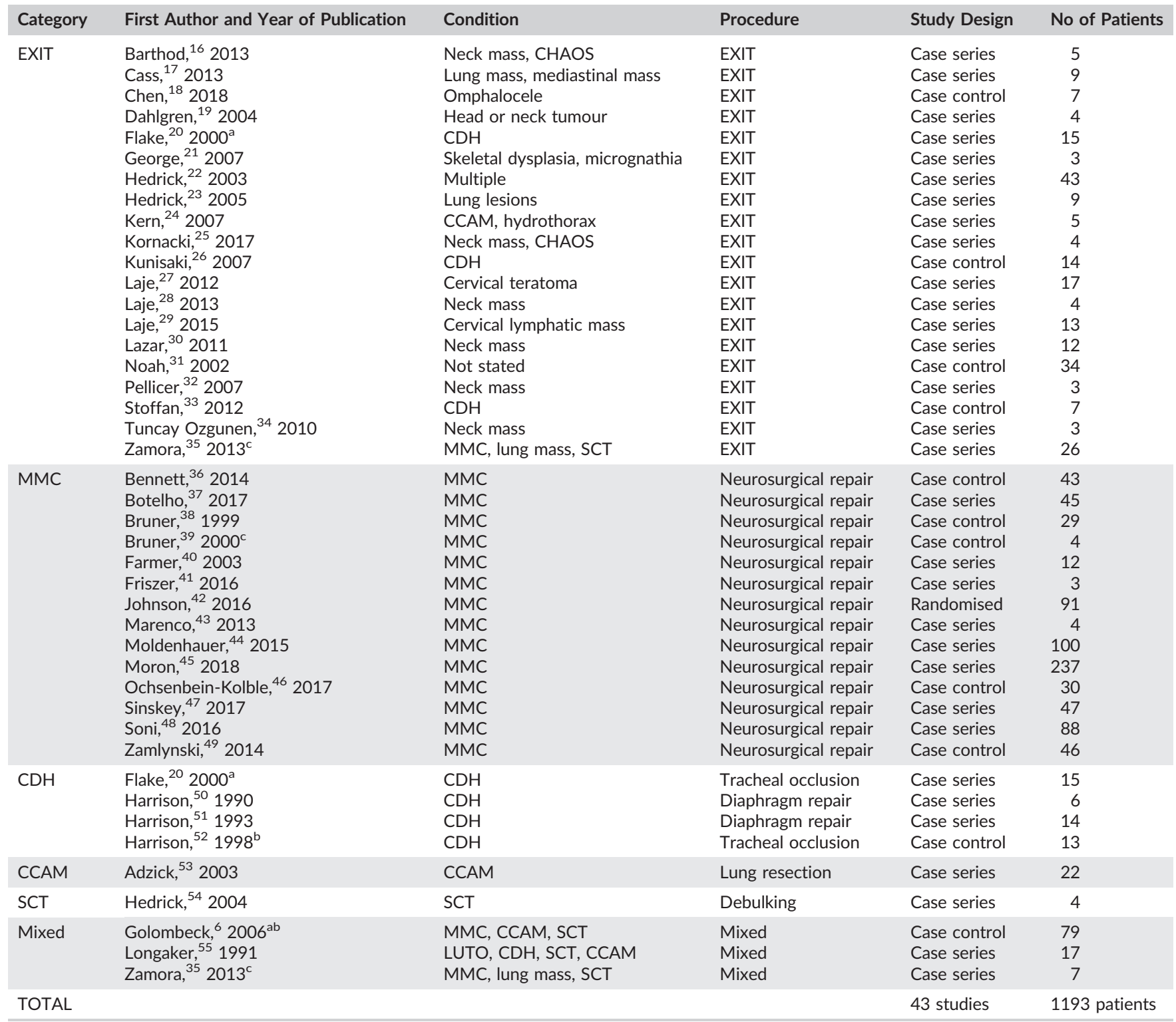

Abbreviations: CCAM, congenital cystic adenomatoid malformation; CD, congenital diaphragmatic hernia; CHAOS, congenital high airway obstruction syndrome; EXIT, ex utero intrapartum treatment; LUTO, lower urinary tract obstruction; MMC, myelomeningocele; SCT, sacrococcygeal teratoma.

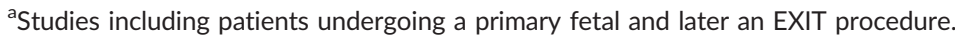

${ }^{b}$ Studies including both open and fetoscopic procedures also included in Table 2.

'Studies including immediate and late complications also included in Table 3.

Haemorrhage severe enough to prompt delivery or termination of pregnancy at the time of surgery as a life-saving procedure for the mother (Clavien-Dindo grade III) occurred in $0.92 \%$ of open fetal (95\% Cl, 0.46-1.62) and $0.26 \%$ of fetoscopic surgeries (95\% Cl, $0.17-$ 0.38). Three cases ${ }^{38,45,46}$ occurred because of placental abruption during open fetal surgery for myelomeningocele (MMC) repair, following which delivery occurred, with all three fetuses surviving. Two cases ${ }^{59,75}$ occurred following laser photocoagulation for TTTS said to be due to "excessive bleeding from placental anastomoses" and the uterine wall, respectively. Two cases ${ }^{119,121}$ occurred during selective reduction, with haemorrhage from the uterine wall prompting delivery.
Finally, one pregnancy was terminated because of bleeding from a trocar placental injury during fetoscopic MMC repair. ${ }^{172}$

In total, placental abruption (Clavien-Dindo grade III) occurred intraoperatively in $1.28 \%$ of open fetal $(95 \% \mathrm{Cl}, 0.73-1.98)$ and in $0.28 \%$ of fetoscopic surgeries $(95 \% \mathrm{Cl}, 0.18-0.39)$. Bleeding during the procedure was noted in $1.97 \%$ of open fetal $(95 \% \mathrm{Cl}, 0.97-3.31)$ and in $1.74 \%$ of fetoscopic surgery cases ( $95 \% \mathrm{Cl}, 1.25-2.32)$. Intraoperative blood transfusion was required in $1.00 \%$ of patients undergoing open fetal surgery $(95 \% \mathrm{Cl}, 0.53-1.64)$ and in $0.27 \%$ undergoing fetoscopic surgery $(95 \% \mathrm{Cl}, 0.18-0.38)$. Intraoperative skin burns at the site of diathermy pads occurred in $0.26 \%$ of patients $(95 \% \mathrm{Cl}$, 
TABLE 2 Included studies of fetoscopic surgery

\begin{tabular}{|c|c|c|c|c|c|}
\hline Category & $\begin{array}{l}\text { First Author and Year } \\
\text { of Publication }\end{array}$ & Condition & Procedure & $\begin{array}{l}\text { Study } \\
\text { Design }\end{array}$ & $\begin{array}{l}\text { No of } \\
\text { Patients }\end{array}$ \\
\hline \multirow{61}{*}{$\begin{array}{l}\text { Multiple pregnancy } \\
\text { complications } \\
\text { treated with laser }\end{array}$} & Aboudiab, ${ }^{56} 2017$ & TTTS & Laser photocoagulation & Case series & 18 \\
\hline & Baschat, $^{57} 2013$ & TTTS & Laser photocoagulation & Case control & 147 \\
\hline & Chalouhi, ${ }^{58} 2016$ & TTTS (triplets) & Laser photocoagulation & Case series & 22 \\
\hline & Chang, $^{59} 2006$ & TTTS & Laser photocoagulation & Case series & 27 \\
\hline & Chang, $^{60} 2016$ & TTTS & Laser photocoagulation & Case control & 100 \\
\hline & Chmait, ${ }^{61} 2013$ & TTTS & Laser photocoagulation & Case control & 318 \\
\hline & Chmait, $^{62} 2017$ & TTTS & Laser photocoagulation & Case series & 19 \\
\hline & Crombleholme, $^{63} 2007$ & TTTS & Laser photocoagulation & Randomised & 20 \\
\hline & De Lia, ${ }^{64} 1995$ & TTTS & Laser photocoagulation & Case series & 26 \\
\hline & De Lia, ${ }^{65} 1999$ & TTTS & Laser photocoagulation & Case series & 67 \\
\hline & De Lia, ${ }^{66} 2009$ & TTTS (triplets) & Laser photocoagulation & Case series & 10 \\
\hline & Deprest, $^{67} 1998$ & TTTS & Laser photocoagulation & Case series & 6 \\
\hline & Draga, $^{68} 2016$ & TTTS & Laser photocoagulation & Case series & 37 \\
\hline & Duron, $^{69} 2014$ & TTTS & Laser photocoagulation & Case control & 85 \\
\hline & $\mathrm{Ek}^{70} 2012$ & TTTS & Laser photocoagulation & Case series & 67 \\
\hline & Habli, $^{71} 2009$ & TTTS & Laser photocoagulation & Case series & 152 \\
\hline & Has, $^{72} 2014$ & TTTS & Laser photocoagulation & Case series & 85 \\
\hline & Hecher, ${ }^{73} 2000$ & TTTS & Laser photocoagulation & Case control & 200 \\
\hline & Hernandez-Andrade, ${ }^{74} 2011$ & TTTS & Laser photocoagulation & Case series & 35 \\
\hline & Huber, ${ }^{75} 2008$ & TTTS & Laser photocoagulation & Case control & 176 \\
\hline & Ishii, ${ }^{76} 2014$ & TTTS (triplets) & Laser photocoagulation & Case series & 16 \\
\hline & Ishii,, 2015 & sFGR & Laser photocoagulation & Case series & 10 \\
\hline & Lanna, $^{78} 2017$ & TTTS & Laser photocoagulation & Case control & 373 \\
\hline & Lecointre, $^{79} 2017$ & TTTS & Laser photocoagulation & Case control & 200 \\
\hline & Malshe, ${ }^{80} 2017$ & TTTS & Laser photocoagulation & Case series & 203 \\
\hline & Martinez, ${ }^{81} 2012$ & TTTS & Laser photocoagulation & Case series & 500 \\
\hline & Middeldorp, ${ }^{82} 2007$ & TTTS & Laser photocoagulation & Case series & 100 \\
\hline & Miyadahira, ${ }^{83} 2018$ & sFGR & Laser photocoagulation & Case control & 67 \\
\hline & Molina-Garcia, ${ }^{84} 2009$ & TTTS, sFGR & Laser photocoagulation & Case series & 22 \\
\hline & Morris, $^{85} 2010$ & TTTS & Laser photocoagulation & Case series & 164 \\
\hline & Mullers, ${ }^{86} 2015$ & TTTS & Laser photocoagulation & Case series & 105 \\
\hline & Nakata, ${ }^{87} 2016$ & TTTS & Laser photocoagulation & Case series & 6 \\
\hline & Nguyen, ${ }^{88} 2012$ & TTTS & Laser photocoagulation & Case series & 98 \\
\hline & Ozawa, ${ }^{89} 2017$ & $\begin{array}{l}\text { Amniotic fluid } \\
\text { discordance }\end{array}$ & Laser photocoagulation & Case series & 11 \\
\hline & Papanna, ${ }^{90} 2010$ & TTTS & Laser photocoagulation & Case control & 48 \\
\hline & Papanna, ${ }^{91} 2012$ & TTTS & Laser photocoagulation & Case control & 163 \\
\hline & Peeters, ${ }^{92} 2014$ & TTTS & Laser photocoagulation & Case control & 338 \\
\hline & Persico, ${ }^{93} 2016$ & TTTS & Laser photocoagulation & Case series & 106 \\
\hline & Quintero, ${ }^{94} 2000$ & TTTS & Laser photocoagulation & Case control & 92 \\
\hline & Quintero, ${ }^{95} 2001$ & sFGR & Laser photocoagulation & Case series & 11 \\
\hline & Rossi, ${ }^{96} 2008$ & TTTS & Laser photocoagulation & Case control & 266 \\
\hline & Ruano, ${ }^{97} 2009$ & TTTS & Laser photocoagulation & Case series & 19 \\
\hline & Ruegg, ${ }^{98} 2018$ & TTTS & Laser photocoagulation & Case control & 37 \\
\hline & Rustico, ${ }^{99} 2012$ & TTTS & Laser photocoagulation & Case series & 150 \\
\hline & Said, ${ }^{100} 2008$ & TTTS & Laser photocoagulation & Case series & 10 \\
\hline & Senat, ${ }^{101} 2004$ & TTTS & Laser photocoagulation & Randomised & 72 \\
\hline & Sepulveda, ${ }^{102} 2007$ & TTTS & Laser photocoagulation & Case series & 33 \\
\hline & Shamshirsaz, ${ }^{103} 2015$ & TTTS & Laser photocoagulation & Case control & 55 \\
\hline & Slaghekke, ${ }^{104} 2014$ & TTTS & Laser photocoagulation & Randomised & 274 \\
\hline & Taniguchi, ${ }^{105} 2015$ & TTTS & Laser photocoagulation & Case series & 3 \\
\hline & Tchirikov, $^{106} 2011$ & TTTS & Laser photocoagulation & Case control & 80 \\
\hline & Teoh, ${ }^{107} 2013$ & TTTS & Laser photocoagulation & Case series & 49 \\
\hline & Thia, ${ }^{108} 2017$ & TTTS & Laser photocoagulation & Case series & 5 \\
\hline & Ville, ${ }^{109} 1997$ & TTTS & Laser photocoagulation & Case series & 132 \\
\hline & Ville, ${ }^{110} 1998$ & TTTS & Laser photocoagulation & Case control & 44 \\
\hline & Weingertner, ${ }^{111} 2011$ & TTTS & Laser photocoagulation & Case series & 100 \\
\hline & Wilson, ${ }^{112} 2016$ & TTTS & Laser photocoagulation & Case series & 151 \\
\hline & Yamamoto, ${ }^{113} 2005$ & TTTS & Laser photocoagulation & Case series & 175 \\
\hline & Yang, ${ }^{114} 2010$ & TTTS & Laser photocoagulation & Case series & 30 \\
\hline & Zaretsky, ${ }^{115} 2018$ & TTTS & Laser photocoagulation & Case series & 749 \\
\hline & Zhao, ${ }^{116} 2016$ & TTTS & Laser photocoagulation & Case control & 62 \\
\hline \multirow{5}{*}{$\begin{array}{l}\text { Multiple pregnancy } \\
\text { complications } \\
\text { treated } \\
\text { with selective } \\
\text { reduction }\end{array}$} & Bebbington, ${ }^{117} 2012$ & $\begin{array}{l}\text { TTTS, TRAP, sFGR, } \\
\text { discordant anomaly }\end{array}$ & RFA & Case control & 146 \\
\hline & Berg, ${ }^{118} 2014$ & TRAP & RFA & Case control & 7 \\
\hline & Delabaere, ${ }^{119} 2013$ & $\begin{array}{l}\text { TTTS, TRAP, sFGR, } \\
\text { discordant anomaly }\end{array}$ & $\begin{array}{l}\text { BCC, cord compression, } \\
\text { cord ligation }\end{array}$ & Case series & 30 \\
\hline & Deprest, ${ }^{120} 2000$ & TTTS, TRAP & $\mathrm{BCC}$ & Case series & 10 \\
\hline & Gallot, $^{121} 2003$ & TTTS, TRAP & $\mathrm{CO}$ & Case series & 11 \\
\hline
\end{tabular}


TABLE 2

(Continued)

\begin{tabular}{|c|c|c|c|c|c|}
\hline Category & $\begin{array}{l}\text { First Author and Year } \\
\text { of Publication }\end{array}$ & Condition & Procedure & $\begin{array}{l}\text { Study } \\
\text { Design }\end{array}$ & $\begin{array}{l}\text { No of } \\
\text { Patients }\end{array}$ \\
\hline & Gouverneur, $^{122} 2009$ & $\begin{array}{l}\text { TTTS, TRAP, sFGR, } \\
\text { discordant anomaly }\end{array}$ & $\begin{array}{l}\text { BCC, laser cord } \\
\text { photocoagulation }\end{array}$ & Case series & 54 \\
\hline & Gul, $^{123} 2008$ & TTTS, TRAP, discordant anomaly & $\mathrm{BCC}$ & Case series & 9 \\
\hline & Has, $^{124} 2014$ & $\begin{array}{l}\text { TTTS, TRAP, sFGR, } \\
\text { discordant anomaly }\end{array}$ & $\mathrm{BCC}$ & Case series & 71 \\
\hline & $\mathrm{He},{ }^{125} 2010$ & $\begin{array}{l}\text { TTTS, TRAP, sFGR, } \\
\text { discordant anomaly }\end{array}$ & $\mathrm{BCC}$ & Case series & 14 \\
\hline & llagan, $^{126} 2008$ & $\begin{array}{l}\text { TTTS, TRAP, } \\
\text { discordant anomaly }\end{array}$ & $\mathrm{BCC}$ & Case series & 27 \\
\hline & Jelin, ${ }^{127} 2010$ & TRAP & RFA & Case control & 7 \\
\hline & King, $^{128} 2017$ & TRAP, discordant anomaly & Laser cord photocoagulation & Case series & 43 \\
\hline & Lanna, $^{129} 2012$ & $\begin{array}{l}\text { TTTS, TRAP, sFGR, } \\
\text { discordant anomaly }\end{array}$ & $\mathrm{BCC}$ & Case series & 118 \\
\hline & Lee, ${ }^{130} 2013$ & TRAP & RFA & Case series & 98 \\
\hline & Lewi, ${ }^{131} 2006$ & $\begin{array}{l}\text { TTTS, TRAP, sFGR, } \\
\text { discordant anomaly }\end{array}$ & Laser cord photocoagulation & Case series & 80 \\
\hline & Moise, ${ }^{132} 2008$ & TTTS, discordant anomaly & RFA & Case series & 9 \\
\hline & Nobili, ${ }^{133} 2013$ & Discordant anomaly & BCC & Case series & 48 \\
\hline & Paramasivam, ${ }^{134} 2010$ & $\begin{array}{l}\text { TTTS, TRAP, sFGR, } \\
\text { discordant anomaly }\end{array}$ & RFA & Case series & 35 \\
\hline & Peng, ${ }^{135} 2016$ & $\begin{array}{l}\text { TTTS, TRAP, sFGR, } \\
\text { discordant anomaly, TAPS }\end{array}$ & $\mathrm{BCC}$ & Case control & 93 \\
\hline & Quintero, ${ }^{136} 1996$ & TTTS, TRAP, discordant anomaly & $\mathrm{CO}$ & Case series & 13 \\
\hline & Quintero, ${ }^{137} 2006$ & TRAP & $\mathrm{CO}$ or laser photocoagulation & Case control & 51 \\
\hline & Roman, $^{138} 2010$ & $\begin{array}{l}\text { TTTS, TRAP, sFGR, } \\
\text { discordant anomaly }\end{array}$ & RFA & Case control & 60 \\
\hline & Schou, ${ }^{139} 2018$ & $\begin{array}{l}\text { TTTS, TRAP, sFGR, } \\
\text { discordant anomaly }\end{array}$ & $\mathrm{BCC}$ & Case control & 102 \\
\hline & Sugibayashi, ${ }^{140} 2016$ & TRAP & RFA & Case series & 40 \\
\hline & Takano, ${ }^{141} 2015$ & TRAP & $\begin{array}{l}\text { Laser photocoagulation } \\
+/- \text { transection of } \\
\text { cord (MCMA) }\end{array}$ & Case series & 10 \\
\hline & Taylor, ${ }^{142} 2002$ & TTTS & $\mathrm{BCC}$ & Case series & 15 \\
\hline & Tsao, ${ }^{143} 2002$ & TRAP & RFA & Case series & 13 \\
\hline & Zhang, $^{144} 2018$ & TRAP & RFA & Case series & 25 \\
\hline \multirow[t]{14}{*}{$\mathrm{CDH}$} & Deprest, ${ }^{145} 2005$ & $\mathrm{CDH}$ & FETO & Case series & 20 \\
\hline & Harrison, ${ }^{52} 1998^{a}$ & $\mathrm{CDH}$ & Tracheal clip & Case control & 8 \\
\hline & Harrison, ${ }^{146} 2003$ & $\mathrm{CDH}$ & FETO & Randomised & 11 \\
\hline & Jani, $^{147} 2005$ & $\mathrm{CDH}$ & FETO & Case series & 24 \\
\hline & Jani, $^{148} 2006$ & $\mathrm{CDH}$ & FETO & Case series & 28 \\
\hline & Jani, $^{149} 2009$ & $\mathrm{CDH}$ & FETO & Case series & 210 \\
\hline & Jimenez, ${ }^{150} 2017$ & $\mathrm{CDH}$ & $\begin{array}{l}\text { Fetoscopic balloon } \\
\text { removal }\end{array}$ & Case control & 201 \\
\hline & Kosinski, ${ }^{151} 2017$ & $\mathrm{CDH}$ & FETO & Case series & 28 \\
\hline & Manrique, ${ }^{152} 2008$ & $\mathrm{CDH}$ & FETO & Case control & 11 \\
\hline & Peralta, ${ }^{153} 2011$ & $\mathrm{CDH}$ & FETO & Case series & 8 \\
\hline & Persico, ${ }^{154} 2017$ & $\mathrm{CDH}$ & FETO & Case series & 21 \\
\hline & Ruano, $^{155} 2012$ & $\mathrm{CDH}$ & FETO & Case control & 35 \\
\hline & Ruano, $^{156} 2012$ & $\mathrm{CDH}$ & FETO & Randomised & 20 \\
\hline & Ruano, ${ }^{157} 2013$ & $\mathrm{CDH}$ & FETO & Case control & 17 \\
\hline \multirow[t]{9}{*}{ MMC } & Arens, ${ }^{158} 2017$ & MMC & Patch & Case series & 59 \\
\hline & Belfort, $^{159} 2017$ & MMC & $\begin{array}{l}\text { Single layer suture } \\
\text { (skin + dura) }\end{array}$ & Case series & 22 \\
\hline & Bruner, $^{39} 2000^{a}$ & MMC & Maternal skin graft & Case control & 4 \\
\hline & Degenhardt, ${ }^{160} 2014$ & MMC & Patch & Case series & 51 \\
\hline & Kohn, ${ }^{161} 2018$ & MMC & Patch & Case series & 34 \\
\hline & Pedreira, ${ }^{162} 2014$ & MMC & Patch + skin suture & Case series & 4 \\
\hline & Pedreira, ${ }^{163} 2016$ & MMC & Patch + skin suture & Case series & 10 \\
\hline & Verbeek, ${ }^{164} 2012$ & MMC & Patch & Case control & 19 \\
\hline & Ziemann, ${ }^{165} 2018$ & MMC & Patch & Case series & 65 \\
\hline \multirow[t]{3}{*}{ LUTO } & Morris, ${ }^{166} 2013$ & LUTO & $\begin{array}{l}\text { Vesicoamniotic } \\
\text { shunting }\end{array}$ & Randomised & 16 \\
\hline & Ruano, ${ }^{167} 2010$ & LUTO & Cystoscopy & Case control & 11 \\
\hline & Welsh, ${ }^{168} 2003$ & LUTO & Cystoscopy & Case series & 13 \\
\hline \multirow[t]{2}{*}{ Shunts } & Cavalheiro, ${ }^{169} 2011$ & Ventriculomegaly & Shunting & Case series & 30 \\
\hline & Mallman, ${ }^{170} 2017$ & Hydrothorax & Shunting & Case series & 78 \\
\hline
\end{tabular}


TABLE 2 (Continued)

\begin{tabular}{|c|c|c|c|c|c|}
\hline Category & $\begin{array}{l}\text { First Author and Year } \\
\text { of Publication }\end{array}$ & Condition & Procedure & $\begin{array}{l}\text { Study } \\
\text { Design }\end{array}$ & $\begin{array}{l}\text { No of } \\
\text { Patients }\end{array}$ \\
\hline Mixed & $\begin{array}{l}\text { Golombeck, }{ }^{6} 2006^{a} \\
\text { Kohl, }{ }^{171} 2006 \\
\text { Kohl, }^{172} 2010 \\
\text { Nivatpumin, }^{173} 2016 \\
\text { Peralta, }^{174}{ }_{2010}\end{array}$ & $\begin{array}{l}\text { TTTS, TRAP, CDH, LUTO } \\
\text { MMC, CDH, CHAOS } \\
\text { MMC, TTTS, CDH, CHAOS, ABS } \\
\text { TTTS, LUTO, CDH, TRAPS } \\
\text { TTTS, CDH, TRAP }\end{array}$ & $\begin{array}{l}\text { Mixed } \\
\text { Mixed } \\
\text { Mixed } \\
\text { Mixed } \\
\text { Mixed }\end{array}$ & $\begin{array}{l}\text { Case control } \\
\text { Case series } \\
\text { Case series } \\
\text { Case series } \\
\text { Case series }\end{array}$ & $\begin{array}{l}99 \\
16 \\
37 \\
152 \\
56\end{array}$ \\
\hline TOTAL & & & & 122 studies & 9403 pat \\
\hline
\end{tabular}

Abbreviations: $\mathrm{BCC}$, bipolar cord coagulation; $\mathrm{CDH}$, congenital diaphragmatic hernia; $\mathrm{CHAOS}$, congenital high airway obstruction syndrome; $\mathrm{CO}$, cord occlusion; FETO, fetoscopic endoluminal tracheal occlusion; LUTO; lower urinary tract obstruction; MCMA, monochorionic monoamniotic; MMC, myelomeningocele; RFA, cord radiofrequency ablation; sFGR; selective fetal growth restriction; TAPS, twin anaemia-polycythaemia sequence; TO, tracheal occlusion; TRAP, twin reversed arterial perfusion sequence; TTTS, twin-to-twin transfusion syndrome.

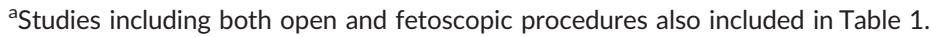

TABLE 3 Included studies of open and fetoscopic surgery focusing on late complications

\begin{tabular}{|c|c|c|c|c|}
\hline $\begin{array}{l}\text { First Author and Year } \\
\text { of Publication }\end{array}$ & Type of Surgery & Condition & Study Design & $\begin{array}{l}\text { Number of } \\
\text { Patients }\end{array}$ \\
\hline Farrell, ${ }^{4} 1999$ & Open & CDH, CCAM, LUTO, SCT, & Case series & 45 \\
\hline Thom, ${ }^{175} 2016$ & Open & MMC & Randomised & 87 \\
\hline Wilson, ${ }^{176} 2010$ & Open & MMC, CCAM, CDH, SCT, mediastinal teratoma & Case series & 47 \\
\hline Zamora, ${ }^{35} 2013^{a}$ & Open & MMC, lung mass, SCT, EXIT & Case series & 33 \\
\hline Gregoir, ${ }^{177} 2016$ & Fetoscopic & $\mathrm{CDH}$ & Case control & 89 \\
\hline Vergote, ${ }^{179} 2018$ & Fetoscopic & TTTS & Case control & 92 \\
\hline TOTAL & & & 7 studies & 515 patients \\
\hline
\end{tabular}

Abbreviations: CCAM, congenital cystic adenomatoid malformation; CDH, congenital diaphragmatic hernia; EXIT, ex utero intrapartum treatment; LUTO, lower urinary tract obstruction; MMC, myelomeningocele; SCT, sacrococcygeal teratoma; TTTS, twin-to-twin transfusion syndrome.

${ }^{\text {a }}$ Studies including immediate and late complications, also included in Table 1.

0.17-0.37) during fetoscopic surgery; this outcome was not reported in any open fetal surgery.

\subsection{Maternal complications in the index pregnancy -postoperative}

One study on laser photocoagulation for TTTS $(n=132)^{110}$ reported a maternal death from disseminated intravascular coagulation (DIC) 4 weeks following an uneventful procedure. A post-mortem examination did not find any evidence of chorioamnionitis or amniotic fluid embolism, and the authors therefore concluded that this death was unrelated to the procedure.

Haemorrhage severe enough to prompt return to theatre for termination or delivery of the pregnancy within 24 hours was not reported following any open fetal surgeries but occurred following $0.25 \%$ of fetoscopic procedures $(95 \% \mathrm{Cl}, 0.16-0.37)$. This included one ${ }^{171} 4$-hour post-fetoscopic tracheal balloon removal with no cause of the bleeding found. There were two late placental abruptions, one $^{113}$ 12-hour post-laser photocoagulation, and one ${ }^{142}$ within 24 hours of bipolar cord coagulation.

Placental abruption occurred in $1.80 \%$ of patients following open fetal (95\% Cl, 1.14-2.63) and in $1.29 \%$ following fetoscopic surgery (95\% Cl, 0.90-1.75). Post-operative blood transfusion was given to
$3.36 \%$ after open fetal surgery $(95 \% \mathrm{Cl}, 1.85-5.29)$ and in $0.32 \%$ following fetoscopic surgery $(95 \% \mathrm{Cl}, 0.22-0.44)$.

Chorioamnionitis following open fetal surgery or endometritis following an EXIT procedure occurred in $4.13 \%$ of women $(95 \% \mathrm{Cl}$, $3.03-5.40)$, and in $1.45 \%$ undergoing fetoscopic surgery $(95 \% \mathrm{Cl}$, 1.06-1.90). Of those, PROM was reported to have occurred in $47.78 \%$ following open fetal surgery $(95 \% \mathrm{Cl}, 23.01-73.16)$ and in $36.31 \%$ following fetoscopic surgery (95\% Cl, 22.00-51.99). One study reported severe chorioamnionitis 5 days after bipolar cord coagulation $^{131}$ with septic shock and acute kidney injury, which resolved leaving $70 \%$ residual renal function. Sepsis was also reported in one patient ${ }^{61}$ with chorioamnionitis following fetoscopic laser photocoagulation and in one patient ${ }^{49}$ following open $\mathrm{MMC}$ repair who developed post-operative peritonitis requiring an emergency laparotomy and delivery. Post-operative pneumonia occurred in two patients-one ${ }^{132}$ following fetoscopic radiofrequency ablation (RFA), necessitating 3 days of intubation and intensive care unit (ICU) care and one requiring ICU admission ${ }^{36}$ following open $\mathrm{MMC}$ repair.

Pulmonary oedema occurred in $4.32 \%$ of open fetal surgery cases (95\% Cl, 2.32-6.90), and in $0.63 \%$ of fetoscopic cases (95\% Cl, $0.43-$ 0.87). Three studies in which post-operative pulmonary oedema occurred reported on perioperative fluid management (3/102, $2.9 \%)$, and 33 reported on the use of magnesium sulphate (33/102, 32.4\%) without specifically suggesting causality. Six women required ICU 
TABLE 4 Maternal complications occurring with open or fetoscopic fetal surgery

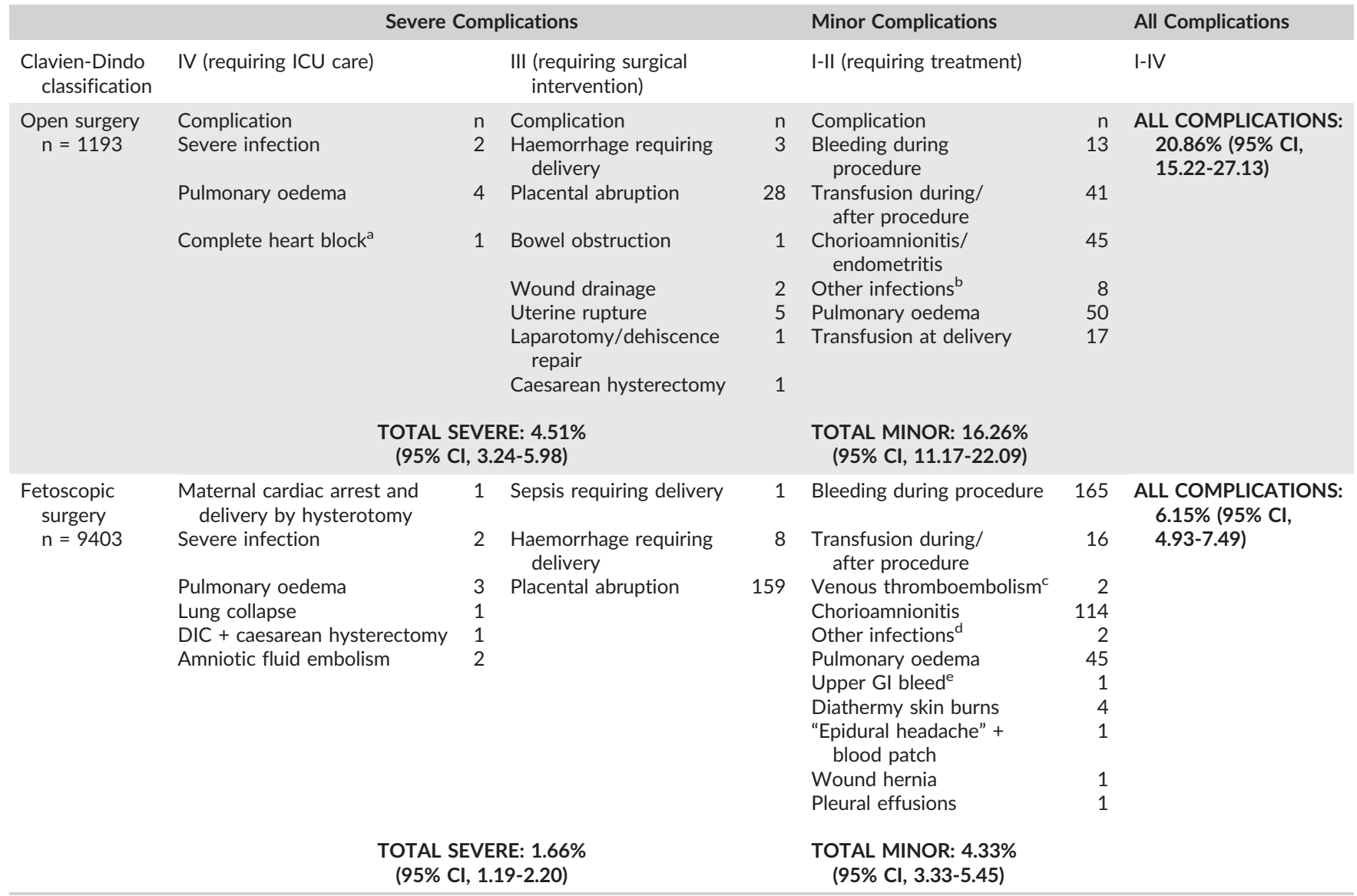

Abbreviations: $\mathrm{Cl}$, confidence interval; $\mathrm{Gl}$, gastrointestinal; $\mathrm{n}$, number of women. Pooled proportions calculated using random effect model for meta-analysis. ${ }^{a}$ Complete heart block considered to be tocolysis-related (magnesium sulphate).

bother infections in open surgery: wound (6), chest (1), urinary tract (1).

'Venous thromboembolism: confirmed pulmonary embolism (1); suspected PE with confirmed deep vein thrombosis (1).

${ }^{\mathrm{d} O t h e r}$ infections in fetoscopic surgery: wound (1), chest (1).

eUpper GI bleed considered to be tocolysis-related (indomethacin).

admission, with four requiring intubation and ventilation; three following open fetal surgery, ${ }^{6,20}$ and three following fetoscopic surgery. $69,87,99$

\section{7 | Maternal complications in the index pregnancy -at delivery}

Only a few fetoscopic surgery studies (4/121 studies, $0.33 \%$ ) reported findings or complications at delivery. Complications at delivery following open fetal surgery are shown in Table 4. Hysterectomy at or around the time of delivery was reported in two patients (ClavienDindo grade III). In one case, ${ }^{44}$ caesarean delivery following open MMC repair in a woman with two previous caesareans, intraabdominal scarring and friable tissue eventually resulted in hysterectomy. In the second case, ${ }^{99}$ following laser photocoagulation for TTTS and PROM, a caesarean section was performed at 33 weeks' gestation. A hysterectomy was eventually required because of haemorrhage with DIC, and the patient spent 5 days in ICU, where she also experienced an iatrogenic pneumothorax.

Uterine rupture occurred in $0.90 \%$ of patients at delivery following open fetal surgery (excluding EXIT procedures) in the index pregnancy $(95 \% \mathrm{Cl}, 0.41-1.59)$, and uterine dehiscence occurred in $3.67 \%$ (95\% Cl, 2.01-5.81). Blood transfusion was given to $1.83 \%$ of women (95\% $\mathrm{Cl}, 1.16-2.65)$ at delivery following open fetal surgery.

\section{8 | Overall maternal complication rates}

Table 4 displays maternal complications. In open fetal surgery, there was a $4.51 \%$ severe $(95 \% \mathrm{Cl}, 3.24-5.98)$, a $16.26 \%$ minor complication rate $(95 \% \mathrm{Cl}, 11.17-22.09)$, and a total complication rate of $20.86 \%$ (95\% Cl, 15.22-27.13). For fetoscopic surgery, the corresponding rates were: $1.66 \%$ severe (95\% Cl, 1.19-2.20), 4.33\% minor (95\% Cl, 3.335.45), and 6.15\% total complications ( $95 \% \mathrm{Cl}, 4.93-7.49)$. Complication rates in the six commonest fetal surgical procedures performed are displayed in Table 5. 
TABLE 5 Maternal complications according to type of fetal surgery in the six most common procedures

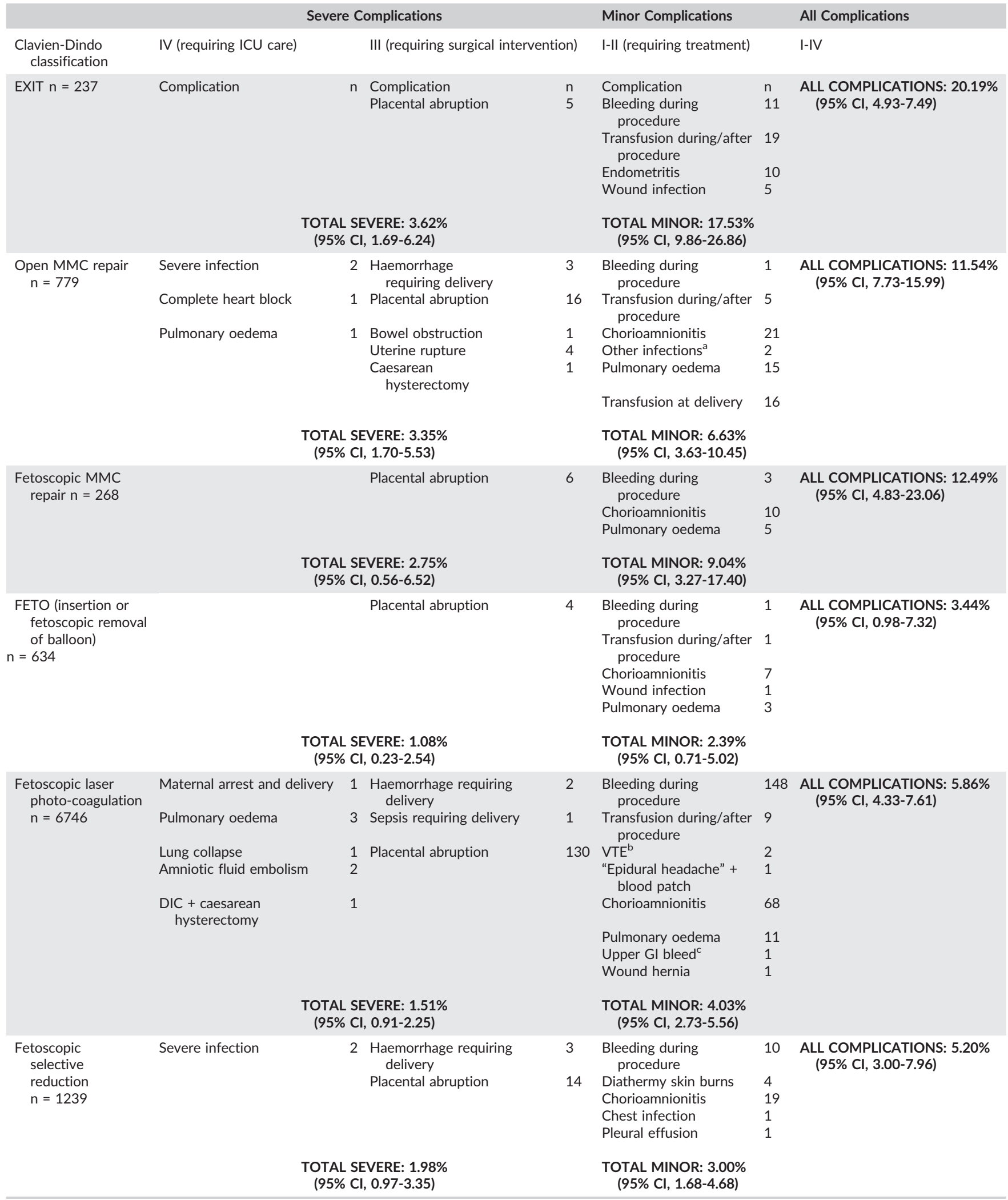

Abbreviations: DIC, disseminated intravascular coagulation; EXIT, ex utero intrapartum treatment; FETO, fetoscopic endoluminal tracheal occlusion; $\mathrm{Gl}$, gastrointestinal; MMC, myelomeningocele; n, number of women. Pooled proportions calculated using random effect model for meta-analysis.

${ }^{\mathrm{a}}$ Other infections in MMC surgery: chest (1), urinary tract (1).

${ }^{b}$ Venous thromboembolism: confirmed pulmonary embolism (1); suspected PE with confirmed deep vein thrombosis (1).

'Upper GI bleed considered to be tocolysis-related (indomethacin). 


\section{9 | Maternal outcomes following the index pregnancy (long-term)}

Table 6 shows subsequent pregnancy outcomes and long-term maternal outcomes following a pregnancy in which fetal surgery was performed. New difficulties in conceiving were described in $3.81 \%$ of women after open fetal surgery $(95 \% \mathrm{Cl}, 1.22-7.76$, reported in four studies); this outcome was not reported to occur after fetoscopic surgery (three studies). Pregnancy loss prior to 24 weeks' gestation occurred in $19.95 \%$ of pregnancies conceived following open fetal surgery $(95 \% \mathrm{Cl}, 13.37-27.48$, three studies) and $13.67 \%$ of pregnancies conceived after fetoscopic surgery $(95 \% \mathrm{Cl}, 9.34-18.68$, three studies). Preterm birth occurred in $20.49 \%$ of pregnancies following open fetal surgery ( $95 \% \mathrm{Cl}, 10.48-32.81$, four studies) and in $2.12 \%$ of pregnancies following fetoscopic surgery $(95 \% \mathrm{Cl}, 0.02-9.01$; three studies). Uterine rupture or dehiscence occurred, respectively, in 6.89\% $(95 \%$ $\mathrm{Cl}, 1.34-16.27$, reported in three studies) and $11.09 \%(95 \% \mathrm{Cl}, 5.34-$ 18.59) of pregnancies following open fetal surgery. None were mentioned in fetoscopy studies.

\section{4 | DISCUSSION}

In this systematic review of the literature, we found an overall complication rate of approximately $21 \%$ for open fetal surgery and $6 \%$ for fetoscopic fetal surgery, of which minor complications occurred in $16 \%$ and $4 \%$ of surgeries, respectively. This maternal complication rate excludes obstetric complications, which may also have occurred (eg, PROM, CMS, preterm labour, and preterm delivery). Additionally, many studies of fetal surgery fail to document maternal complications. Out of 751 full-text articles reviewed, 175 (23.3\%) were excluded as no maternal outcomes were stated. Although 68 of these studies focused on a specific aspect of the surgery or its neonatal outcome, 107 studies (92 fetoscopic and 15 open) involving over 9000 patients did not comment on the presence or absence of any complications specifically affecting the mother's health. Often, the "maternal outcomes" stated meant in reality obstetric outcomes (eg, PROM and preterm labour). We also found that maternal complications were often presented from the fetal perspective (eg, fetal demise caused by placental abruption). Thirty included studies (18.1\%) contained a statement that no adverse maternal outcomes were observed without specifying what was meant by maternal outcomes. Among these studies were some large series, including a study of 201 patients undergoing fetoscopic tracheal balloon removal ${ }^{150}$ and studies of $200^{73}$ and $500^{81}$ patients undergoing fetoscopic laser coagulation. It is unlikely that such large numbers of procedures had no maternal complications, and more likely that complications were either not perceived as serious, not reported and/or the patient follow-up was incomplete. This lack of reporting has most likely led to an underestimation of the actual risk of maternal complications in our meta-analysis. Conversely, when maternal complications were reported, there was a wide variability in which outcomes were discussed and how they were presented.

There was a severe complication rate (Clavien-Dindo grade III or IV) of $4.5 \%$ in women undergoing open fetal surgery and $1.7 \%$ undergoing fetoscopic surgery. This is in keeping with a previous multicentre review of maternal complications following laser photocoagulation for TTTS, ${ }^{7}$ which found a $1.0 \%$ rate of severe complications and a $5.4 \%$ total rate of complications across all studies; however, when the authors only included studies, which systematically assessed maternal complications as a primary or secondary outcome, this rose to $1.8 \%$ for severe and $17.4 \%$ for all complications.

In almost all studies of fetal surgery reviewed, long-term maternal follow up was not described. The seven studies that did so had a wide variation in the parameters described. Fertility does not appear to be negatively affected by fetal surgery, with the rates of de novo difficulties for conceiving in this review (3.81\% following open fetal surgery and none following fetoscopic surgery) being comparable, if not less, than published rates of secondary infertility in the general population. ${ }^{180}$ Similarly, the rates of miscarriage described (19.85\% following

TABLE 6 Long-term maternal complications following open and fetoscopic fetal surgery

\begin{tabular}{|c|c|c|c|}
\hline & & $\begin{array}{l}\text { Open Surgery }{ }^{a} \\
\%(95 \% \mathrm{Cl})\end{array}$ & $\begin{array}{l}\text { Fetoscopic Surgery } \\
\%(95 \% \mathrm{Cl})\end{array}$ \\
\hline Conception & $\begin{array}{l}\text { Women attempting further pregnancy } \\
\text { Women conceiving further pregnancy } \\
\text { New sub-fertility }\end{array}$ & $\begin{array}{c}50.11(21.55-78.63) \\
48.33(26.74-70.26) \\
3.81(1.22-7.76)\end{array}$ & $\begin{array}{l}51.76(18.63-84.03) \\
48.20(31.46-65.16) \\
\text { NR }\end{array}$ \\
\hline Pregnancy outcomes & $\begin{array}{l}\text { Miscarriage } \\
\text { Preterm delivery } \\
\text { Uterine rupture } \\
\text { Uterine dehiscence } \\
\text { Excessive bleeding at delivery }\end{array}$ & $\begin{aligned} 19.95 & (13.37-27.48) \\
20.49 & (10.48-32.81) \\
6.89 & (1.34-16.27) \\
11.09 & (5.34-18.59) \\
6.84 & (2.16-13.88)\end{aligned}$ & $\begin{array}{l}13.67(9.34-18.68) \\
2.12(0.02-9.01) \\
0 \\
\text { NR } \\
5.52(2.83-9.03)\end{array}$ \\
\hline Nonpregnancy & $\begin{array}{l}\text { Abdominal pain } \\
\text { Abnormal menstrual bleeding } \\
\text { Gynaecological surgeryc } \\
\text { Psychological symptoms }\end{array}$ & $\begin{array}{l}6.38^{\mathrm{b}} \\
\text { NR } \\
8.68(1.81-19.96) \\
9.09^{\mathrm{b}}\end{array}$ & $\begin{array}{l}9.01(3.84-16.06) \\
6.54(3.43-10.57) \\
\text { NR } \\
32.56(7.70-64.58)\end{array}$ \\
\hline
\end{tabular}

Abbreviation: NR, not reported. Pooled proportions calculated using random effect model for meta-analysis.

${ }^{a}$ Variable denominator as not all outcomes were reported by all studies.

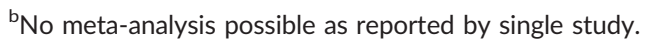

${ }^{\mathrm{C} G y n a e c o l o g i c a l ~ s u r g e r y ~ f o l l o w i n g ~ o p e n ~ f e t a l ~ s u r g e r y: ~ e n d o m e t r i a l ~ a b l a t i o n ~(1), ~ h y s t e r e c t o m y ~(6): ~ c a e s a r e a n ~ h y s t e r e c t o m y ~(1), ~ o v a r i a n ~ c y s t s+/-m e n s t r u a l ~}$ disorder (2), fibroids (1), unknown reason (2). 
open fetal and $13.67 \%$ following fetoscopic surgery) are similar to rates of spontaneous miscarriage in women who have not undergone fetal surgery. ${ }^{181-183}$ Epidemiological studies ${ }^{184}$ have suggested a worldwide preterm birth rate of $11.1 \%$ with a rate of $8.6 \%$ in "developed regions." ${ }^{184}$ In the United States and United Kingdom, it is estimated at $9.8 \%{ }^{185}$ and $7.3 \%,{ }^{186}$ respectively. The preterm birth rate in this review following open fetal surgery $(20.49 \%)$ is higher than the usual prevalence, but not higher following fetoscopic surgery (2.12\%). Open fetal surgery was followed by uterine rupture or dehiscence in $6.89 \%$ and $11.09 \%$ of subsequent pregnancies, respectively, which is in line with published rates of rupture (6.2\%) and dehiscence (12.5\%) following a classical caesarean section. ${ }^{187}$ Conversely, no uterine ruptures were reported following fetoscopic surgery.

This study included the commonest fetal procedures and, from a maternal perspective, involved similar surgical manipulations yet variable operating times. We included studies from multiple centres worldwide and attempted to identify the non-English literature. It is therefore likely that these results are generalisable to fetal surgery performed outside the included studies. An obvious weakness of this systematic review is that most studies did not include a control group. Furthermore, we decided to pool data for meta-analysis despite having high heterogeneity in some results. Another weakness is the extraction of patient data from papers, which is prone to error given the variable reporting; it is possible that some patients had more than one complication and this was not noted or cumulative rates were as a consequence miscalculated.

This systematic review has identified a significant rate of maternal complications, which should be discussed with patients before embarking on fetal surgery. Large studies allow an estimation of the likelihood of these events, insomuch as the cases in these series are unselected and consecutive. Our systematic review search strategy may have missed relevant yet rare complications. For example, a letter to a journal editor describing maternal convulsions during general anaesthesia ${ }^{188}$ was excluded as a case report according to our criteria. In this circumstance, it appears that the patient was also part of the cohort of a study that was included, ${ }^{47}$ but it is possible that other rare events published as case reports have been missed. An international, prospective registry of fetal and fetoscopic surgery, such as the Eurofoetus $^{189}$ and NAFTNet ${ }^{190}$ registries, would be the best way to accurately determine complication types and rates and avoid missing rare complications.

\section{5 | CONCLUSION}

The maternal risks of fetal surgery are accepted by many patients and health care professionals for the possible benefit to the fetus. ${ }^{191,192}$ This systematic review finds that studies of fetal surgery focus on the fetal outcomes of the procedure, and many fail to describe maternal complications. Fetal surgery comes at a risk to the mother, which may be underestimated by fetal therapists because of under-reporting and variable reporting quality. In order to properly quantify maternal risks, outcomes should be reported consistently across all studies of fetal surgery, preferentially in prospective registries.

\section{CONFLICT OF INTEREST}

All authors report no conflict of interest.

\section{FUNDING INFORMATION}

This research is funded by the Wellcome Trust (WT101957) and Engineering and Physical Sciences Research Council (ESPRC) (NS/A000027/1). J.D. is also funded by the Great Ormond Street Hospital Children's Charity Fund. A.L.D. is supported by the National Institute for Health Research University College London Hospitals Biomedical Research Centre. LvdV is funded with support of the Erasmus + Programme of the European Union (Framework Agreement number: 2013-0040). This publication reflects the views only of the author, and the commission cannot be held responsible for any use, which may be made of the information contained therein.

\section{ORCID}

Adalina Sacco (D) https://orcid.org/0000-0002-9182-9628

Tim Van Mieghem (1D https://orcid.org/0000-0002-3034-6905

Anna L. David (D) https://orcid.org/0000-0002-0199-6140

Jan Deprest (iD https://orcid.org/0000-0002-4920-945X

\section{REFERENCES}

1. Deprest JA, Flake AW, Gratacos E, et al. The making of fetal surgery. Prenat Diagn. 2010;30(7):653-667. https://doi.org/10.1002/pd.2571

2. Jancelewicz T, Harrison MR. A history of fetal surgery. Clin Perinatol. 2009;36(2):227. https://doi.org/10.1016/j.clp.2009.03.007-236

3. Deprest JA, Done E, Van Mieghem T, Gucciardo L. Fetal surgery for anesthesiologists. Curr Opin Anaesthesiol. 2008;21(3):298-307. https://doi.org/10.1097/ACO.0b013e3282ff8607

4. Farrell JA, Albanese CT, Jennings RW, Kilpatrick SJ, Bratton BJ, Harrison MR. Maternal fertility is not affected by fetal surgery. Fetal Diagn Ther. 1999;14(3):190-192.

5. Moon-Grady A, Baschat A, Cass D. Fetal treatment 2017: the evolution of fetal therapy centers-a joint opinion from the international fetal medicine and surgical society (IFMSS) and the north American fetal therapy network (NAFTNet). Fetal Diagn Ther. 2017;42(4):241-248.

6. Golombeck K, Ball RH, Lee $\mathrm{H}$, et al. Maternal morbidity after maternal-fetal surgery. Am J Obstet Gynecol. 2006;194(3):834-839.

7. Merz W, Tchatcheva K, Gembruch U, Kohl T. Maternal complications of fetoscopic laser photocoagulation (FLP) for treatment of twin-twin transfusion syndrome (TTTS). J Perinat Med. 2010;38(4):439-443. https://doi.org/10.1515/JPM.2010.061

8. Moher D, Liberati A, Tetzlaff J, Altman D, The PRISMA Group. Preferred reporting items for systematic reviews and meta-analyses: the PRISMA statement. PLoS Med. 2009;6(7):e1000097.

9. Carey TS, Boden SD. A critical guide to case series reports. Spine (Phila Pa 1976). 2003;28(15):1631-1634. https://doi.org/10.1097/ 01.BRS.0000083174.84050.E5

10. The Royal College of Obstetricians and Gynaecologists. Antepartum Haemorrhage Green-top Guideline No. 63. 2011;(63). https://www. rcog.org.uk/globalassets/documents/guidelines/gtg_63.pdf.

11. Gulmezoglu AM, Souza JP, Mathai M. WHO recommendations for the prevention and treatment of postpartum haemorrhage. 2012. https://doi.org/10.1016/j.ijgo.2013.06.024.

12. Dindo D, Demartines N, Clavien PA. Classification of surgical complications: a new proposal with evaluation in a cohort of 6336 
patients and results of a survey. Ann Surg. 2004;240(2):205-213. https://doi.org/10.1097/01.sla.0000133083.54934.ae

13. Higgins J, Green S. Assessing risk of bias in included studies. Cochrane Handb Syst Rev Interv. 2008:187-241.

14. Wells G, Shea B, O'Connell D, Peterson J, Welcho V, Losos M. The Newcastle-Ottawa scale (NOS) for assessing the quality of nonrandomized studies in meta-analyses. Ottawa Heal Res Inst 2011. http://www.ohri.ca/programs/clinical_epidemiology/nos_manual.pdf

15. National Institutes of Health: study quality assessment tools. www.nhlbi. nih.gov/health-topics/study-quality-assessment-tools. Published 2014.

16. Barthod G, Teissier N, Bellarbi N, et al. Fetal airway management on placental support: limitations and ethical considerations in seven cases. J Obstet Gynaecol. 2013;33(8):787-794. https://doi.org/ 10.3109/01443615.2013.823924

17. Cass DL, Olutoye OO, Cassady $\mathrm{Cl}$, et al. EXIT-to-resection for fetuses with large lung masses and persistent mediastinal compression near birth. J Pediatr Surg. 2013;48(1):138-144. https://doi.org/10.1016/ j.jpedsurg.2012.10.067

18. Chen XY, Yang JX, Zhang HY, et al. Ex utero intrapartum treatment for giant congenital omphalocele. World J Pediatr. 2018;(0123456789);14(4):1-5. https://doi.org/10.1007/s12519018-0129-7

19. Dahlgren G, Törnberg DC, Pregner K, Irestedt L. Four cases of the ex utero intrapartum treatment (EXIT) procedure: anesthetic implications. Int J Obstet Anesth. 2004;13(3):178-182.

20. Flake AW, Crombleholme TM, Johnson MP, Howell LJ, Adzick NS. Treatment of severe congenital diaphragmatic hernia by fetal tracheal occlusion: clinical experience with fifteen cases. Am J Obstet Gynecol. 2000;183(5):1059-1066.

21. George RB, Melnick AH, Rose EC, Habib AS. Case series: combined spinal epidural anesthesia for cesarean delivery and ex utero intrapartum treatment procedure. Can J Anaesth. 2007;54(3):218-222.

22. Hedrick HL. Ex utero intrapartum therapy. Semin Pediatr Surg. 2003;12(3):190-195.

23. Hedrick HL, Flake AW, Crombleholme TM, et al. The ex utero intrapartum therapy procedure for high-risk fetal lung lesions. J Pediatr Surg. 2005;40(6):1038-1044. https://doi.org/10.1016/ j.jpedsurg.2005.03.024

24. Kern C, Ange M, Morales PB, Pfister RE. Ex utero intrapartum treatment (EXIT), a resuscitation option for intra-thoracic foetal pathologies. Swiss Med Wkly. 2007;137(19-20):279-285.

25. Kornacki J, Szydłowski J, Skrzypczak J, et al. Use of ex utero intrapartum treatment procedure in fetal neck and high airway anomalies-report of four clinical cases. J Matern Neonatal Med. 2017;0(0);32(5):1-5. https://doi.org/10.1080/14767058.2017.1390740

26. Kunisaki SM, Barnewolt CE, Estroff JA, et al. Ex utero intrapartum treatment with extracorporeal membrane oxygenation for severe congenital diaphragmatic hernia. J Pediatr Surg. 2007;42(1):98.

27. Laje P, Johnson MP, Howell $\sqcup$, et al. Ex utero intrapartum treatment in the management of giant cervical teratomas. J Pediatr Surg. 2012;47(6):1208-1216. https://doi.org/10.1016/j.jpedsurg.2012.03.027

28. Laje P, Howell LJ, Johnson MP, Hedrick HL, Flake AW, Adzick NS. Perinatal management of congenital oropharyngeal tumors: the ex utero intrapartum treatment (EXIT) approach. J Pediatr Surg. 2013;48(10):2005-2010. https://doi.org/10.1016/j.jpedsurg.2013. 02.031

29. Laje P, Peranteau WH, Hedrick HL, et al. Ex utero intrapartum treatment (EXIT) in the management of cervical lymphatic malformation. J Pediatr Surg. 2015;50(2):311-314. https://doi.org/10.1016/j.jpedsurg. 2014.11.024
30. Lazar DA, Olutoye OO, Moise KJ, et al. Ex-utero intrapartum treatment procedure for giant neck masses--fetal and maternal outcomes. J Pediatr Surg. 2011;46(5):817-822. https://doi.org/ 10.1016/j.jpedsurg.2011.02.006

31. Noah MMS, Norton ME, Sandberg P, Esakoff T, Farrell J, Albanese CT. Short-term maternal outcomes that are associated with the EXIT procedure, as compared with cesarean delivery. Am J Obstet Gynecol. 2002;186(4):773-777.

32. Pellicer M, Pumarola F, Peiró JL, et al. EXIT procedure in the management of severe foetal airway obstruction. The paediatric otolaryngologist's perspective. Acta Otorrinolaringol Esp. 2007;58(10):487-490.

33. Stoffan AP, Wilson JM, Jennings RW, Wilkins-Haug LE, Buchmiller TL. Does the ex utero intrapartum treatment to extracorporeal membrane oxygenation procedure change outcomes for high-risk patients with congenital diaphragmatic hernia? J Pediatr Surg. 2012;47(6):1053-1057. https://doi.org/10.1016/j.jpedsurg.2012. 03.004

34. Tuncay Ozgunen F, Kucukgoz Gulec U, Evruke I, Agcabay C, Kadayifci T, Ozcan K. Fetal orofarengeal tumorler ve EXIT islemine dogum hekimi acisindan bakis [the point of view by obstetricians to fetal oropharengal tumors and EXIT procedure: case report]. Turkiye Klin Jinekoloji Obstet. 2010;20(4):247-251.

35. Zamora IJ, Ethun CG, Evans LM, et al. Maternal morbidity and reproductive outcomes related to fetal surgery. J Pediatr Surg. 2013;48(5):951-955. https://doi.org/10.1016/j.jpedsurg.2013.02.010

36. Bennett KA, Carroll MA, Shannon $\mathrm{CN}$, et al. Reducing perinatal complications and preterm delivery for patients undergoing in utero closure of fetal myelomeningocele: further modifications to the multidisciplinary surgical technique. J Neurosurg Pediatr. 2014; 14(1):108-114. https://doi.org/10.3171/2014.3.PEDS13266

37. Botelho R, Imada V, Rodrigues da Costa K, Watanabe L, Rossi RJ. Fetal myelomeningocele repair through a mini-hysterotomy. Fetal Diagn Ther. 2017;42(1):28-34.

38. Bruner JP, Tulipan N, Paschall RL, et al. Fetal surgery for myelomeningocele and the incidence of shunt-dependent hydrocephalus. JAMA. 1999;282(19):1819-1825.

39. Bruner JP, Tulipan NB, Richards WO, Walsh WF, Boehm FH, Vrabcak EK. In utero repair of myelomeningocele: a comparison of endoscopy and hysterotomy. Fetal Diagn Ther. 2000;15(2):83-88.

40. Farmer DL, von Koch CS, Peacock WJ, et al. In utero repair of myelomeningocele: experimental pathophysiology, initial clinical experience, and outcomes. Arch Surg. 2003;138(8):872-878. http://www.hlisd.org/LibraryDetail.aspx?LibraryID=3866

41. Friszer S, Dhombres F, Di Rocco F, et al. Preliminary results from the French study on prenatal repair for fetal myelomeningoceles (the PRIUM study). J Gynecol Obstet Biol Reprod. 2016;45(7):738-744. https://doi.org/10.1016/j.jgyn.2015.09.002

42. Johnson MP, Bennett KA, Rand $L$, et al. The management of myelomeningocele study: obstetrical outcomes and risk factors for obstetrical complications following prenatal surgery. Am J Obstet Gynecol. 2016;215(6):778. https://doi.org/10.1016/j.ajog.2016. 07.052

43. Marenco ML, Márquez J, Ontanilla A, et al. Intrauterine myelomeningocele repair: experience of the fetal medicine and therapy program of the Virgen de Rocío University hospital. Rev Esp Anestesiol Reanim. 2013;60(1):47-53. https://doi.org/10.1016/ j.redar.2012.07.011

44. Moldenhauer JS, Soni S, Rintoul NE, et al. Fetal myelomeningocele repair: the post-MOMS experience at the Children's Hospital of Philadelphia. Fetal Diagn Ther. 2015;37(3):235-240. https://doi.org/ 10.1159/000365353 
45. Moron A, Barbosa M, Milani $\mathrm{H}$, et al. Perinatal outcomes after open fetal surgery for myelomeningocele repair: a retrospective cohort study. BJOG. 2018; 125(10):1280-1286. [Epub ahea. https:// doi.org/10.1111/1471-0528.15312

46. Ochsenbein-Kölble N, Krähenmann F, Hüsler M, et al. Tocolysis for in utero surgery: atosiban performs distinctly better than magnesium sulfate. Fetal Diagn Ther. 2017. https://doi.org/10.1159/ 000478261;44(1):59-64.

47. Sinskey JL, Rollins MD, Whitlock E, et al. Incidence and management of umbilical artery flow abnormalities during open fetal surgery. Fetal Diagn Ther. 2017. https://doi.org/10.1159/000477963;43(4): 274-283.

48. Soni S, Moldenhauer JS, Spinner SS, et al. Chorioamniotic membrane separation and preterm premature rupture of membranes complicating in utero myelomeningocele repair. Am J Obstet Gynecol. 2016;214(5):647. https://doi.org/10.1016/j.ajog.2015.12.003

49. Zamlynski J, Olejek A, Tomasz K. Comparison of prenatal and postnatal treatments of spina bifida in Poland-a non-randomized, single-center study. J Matern Neonatal Med. 2014;27(14):1409-1417.

50. Harrison MR, Langer JC, Adzick NS, et al. Correction of congenital diaphragmatic hernia in utero, V. Initial clinical experience. J Pediatr Surg. 1990;25(1):47-57.

51. Harrison MR, Adzick NS, Flake AW, et al. Correction of congenital diaphragmatic hernia in utero: VI. Hard-earned lessons. J Pediatr Surg. 1993;28(10):1411-1418.

52. Harrison MR, Mychaliska GB, Albanese CT, et al. Correction of congenital diaphragmatic hernia in utero IX: fetuses with poor prognosis (liver herniation and low lung-to-head ratio) can be saved by fetoscopic temporary tracheal occlusion. J Pediatr Surg. 1998;33(7):1017-1023.

53. Adzick NS, Flake AW, Crombleholme TM. Management of congenital lung lesions. Semin Pediatr Surg. 2003;12(1):10-16.

54. Hedrick HL, Flake AW, Crombleholme TM, et al. Sacrococcygeal teratoma: prenatal assessment, fetal intervention, and outcome. J Pediatr Surg. 2004;39(3):430-438.

55. Longaker MT, Golbus MS, Filly RA, Rosen MA, Chang SW, Harrison MR. Maternal outcome after open fetal surgery. A review of the first 17 human cases. JAMA. 1991;265(6):737-741. http://www.hlisd.org/ LibraryDetail.aspx?LibraryID=3866

56. Aboudiab MS, Chon AH, Korst LM, Llanes A, Ouzounian JG, Chmait $\mathrm{RH}$. Management of twin-twin transfusion syndrome with an extremely short cervix. J Obstet Gynaecol. 2018;38(3):359-362. https://doi.org/10.1080/01443615.2017.1330324

57. Baschat AA, Barber J, Pedersen N, Turan OM, Harman CR. Outcome after fetoscopic selective laser ablation of placental anastomoses vs equatorial laser dichorionization for the treatment of twin-to-twin transfusion syndrome. Am J Obstet Gynecol. 2013;209(3):234. https://doi.org/10.1016/j.ajog.2013.05.034

58. Chalouhi GE, Quibel T, Benzina N, Bernard J-P, Essaoui M, Ville Y. Outcome of triplet pregnancies managed for twin-to-twin transfusion syndrome: A single center experience. J Gynecol Obstet Biol Reprod. 2016;45(8):929-935. https://doi.org/10.1016/j.jgyn. 2015.08.006

59. Chang J, Tracy TF, Carr SR, Sorrells DL, Luks FI. Port insertion and removal techniques to minimize premature rupture of the membranes in endoscopic fetal surgery. J Pediatr Surg. 2006;41(5):905-909.

60. Chang Y-L, Chao A-S, Chang S-D, et al. Outcome of twin-twin transfusion syndrome treated by laser therapy in Taiwan's single center: role of Quintero staging system. Taiwan J Obstet Gynecol. 2016;55(5):700-704. https://doi.org/10.1016/j.tjog.2015.05.006
61. Chmait RH, Korst LM, Llanes A, Mullin P, Lee RH, Ouzounian JG. Perioperative characteristics associated with preterm birth in twin-twin transfusion syndrome treated by laser surgery. Am J Obstet Gynecol. 2013;209(3):264. https://doi.org/10.1016/j.ajog.2013.05.025

62. Chmait RH, Kontopoulos EV, Chon AH, Korst LM, Llanes A, Quintero RA. Amniopatch treatment of iatrogenic preterm premature rupture of membranes (iPPROM) after fetoscopic laser surgery for twin-twin transfusion syndrome. J Matern Neonatal Med. 2017;30(11):1349-1354. https://doi.org/10.1080/14767058.2016.1214123

63. Crombleholme TM, Shera D, Lee H, et al. A prospective, randomized, multicenter trial of amnioreduction vs selective fetoscopic laser photocoagulation for the treatment of severe twin-twin transfusion syndrome. Am J Obstet Gynecol. 2007;197(4):396.

64. De Lia JE, Kuhlmann RS, Harstad TW, Cruikshank DP. Fetoscopic laser ablation of placental vessels in severe previable twin-twin transfusion syndrome. Am J Obstet Gynecol. 1995;172(4):1202.

65. De Lia JE, Kuhlmann RS, Lopez KP. Treating previable twin-twin transfusion syndrome with fetoscopic laser surgery: outcomes following the learning curve. J Perinat Med. 1999;27(1):61-67.

66. De Lia JE, Worthington D, Carr MH, Graupe MH, Melone PJ. Placental laser surgery for severe previable feto-fetal transfusion syndrome in triplet gestation. Am J Perinatol. 2009;26(8):559-564. https://doi.org/10.1055/s-0029-1220789

67. Deprest JA, Van Schoubroeck D, Van Ballaer PP, Flageole H, Van Assche FA, Vandenberghe $\mathrm{K}$. Alternative technique for $\mathrm{Nd}$ : YAG laser coagulation in twin-to-twin transfusion syndrome with anterior placenta. Ultrasound Obstet Gynecol. 1998;11(5):347-352.

68. Draga E, Janiak K, Bielak A, et al. Fetal therapy-laser therapy of twin-to-twin transfusion syndrome/TTTS/. Polish Gynaecol. 2016;87(02):104-110. https://doi.org/10.17772/gp/61328

69. Duron VD, Watson-Smith D, Benzuly SE, et al. Maternal and fetal safety of fluid-restrictive general anesthesia for endoscopic fetal surgery in monochorionic twin gestations. J Clin Anesth. 2014;26(3):184-190. https://doi.org/10.1016/j.jclinane.2013.10.010

70. Ek S, Kublickas M, Bui T-H, et al. Establishing a national program for fetoscopic guided laser occlusion for twin-to-twin transfusion syndrome in Sweden. Acta Obstet Gynecol Scand. 2012;91(10): 1196-1200. https://doi.org/10.1111/j.1600-0412.2012.01447.x

71. Habli M, Bombrys A, Lewis D, et al. Incidence of complications in twin-twin transfusion syndrome after selective fetoscopic laser photocoagulation: a single-center experience. Am J Obstet Gynecol. 2009;201(4):417. https://doi.org/10.1016/j.ajog.2009.07.046

72. Has R, Kalelioglu I, Corbacioglu Esmer A, et al. Stage-related outcome after fetoscopic laser ablation in twin-to-twin transfusion syndrome. Fetal Diagn Ther. 2014;36(4):287-292. https://doi.org/10.1159/ 000362385

73. Hecher K, Diehl W, Zikulnig L, Vetter M, Hackelöer BJ. Endoscopic laser coagulation of placental anastomoses in 200 pregnancies with severe mid-trimester twin-to-twin transfusion syndrome. Eur J Obstet Gynecol Reprod Biol. 2000;92(1):135-139.

74. Hernández-Andrade E, Guzmán-Huerta M, Benavides-Serralde JA, et al. Laser ablation of the placental vascular anastomoses for the treatment of twin-to-twin transfusion syndrome. Rev Invest Clin. 2011;63(1):46-52.

75. Huber A, Baschat AA, Bregenzer T, et al. Laser coagulation of placental anastomoses with a 30 degrees fetoscope in severe mid-trimester twin-twin transfusion syndrome with anterior placenta. Ultrasound Obstet Gynecol. 2008;31(4):412-416. https://doi.org/10.1002/uog.5283

76. Ishii K, Nakata M, Wada S, Hayashi S, Murakoshi T, Sago H. Perinatal outcome after laser surgery for triplet gestations with feto-fetal 
transfusion syndrome. Prenat Diagn. 2014;34(8):734-738. https://doi. org/10.1002/pd.4357

77. Ishii K, Nakata M, Wada S, Murakoshi T, Sago H. Feasibility and preliminary outcomes of fetoscopic laser photocoagulation for monochorionic twin gestation with selective intrauterine growth restriction accompanied by severe oligohydramnios. J Obstet Gynaecol Res. 2015;41(11):1732-1737. https://doi.org/10.1111/jog.12827

78. Lanna MM, Faiola S, Consonni D, Rustico MA. Increased risk of placental abruption after Solomon laser treatment of twin-twin transfusion syndrome. Placenta. 2017;53:54-56. https://doi.org/ 10.1016/j.placenta.2017.03.018

79. Lecointre L, Sananès N, Weingertner AS, et al. Photocoagulation laser par fœtoscopie pour syndrome transfuseur-transfusé: analyse d'une série consécutive unicentrique de 200 cas. J Gynecol Obstet Hum Reprod. 2017;46(2):175-181. https://doi.org/10.1016/ j.jogoh.2016.10.004

80. Malshe A, Snowise S, Mann LK, et al. Preterm delivery after fetoscopic laser surgery for twin-twin transfusion syndrome: etiology and risk factors. Ultrasound Obstet Gynecol. 2017;49(5):612-616. https://doi.org/10.1002/uog.15972

81. Martínez JM, Eixarch E, Crispi F, Puerto B, Gratacós E. Tratamiento por fetoscopia de la transfusión feto-fetal: resultados en 500 casos consecutivos. Diagnostico Prenat. 2012;23(3):102-108. https://doi.org/ 10.1016/j.diapre.2012.06.010

82. Middeldorp JM, Sueters M, Lopriore E, et al. Fetoscopic laser surgery in 100 pregnancies with severe twin-to-twin transfusion syndrome in the Netherlands. Fetal Diagn Ther. 2007;22(3):190-194.

83. Miyadahira M, Brizot M, Carvalho M, et al. Type II and III selective fetal growth restriction: perinatal outcomes of expectant management and laser ablation of placental vessels. Clinics. 2018;73:1-5. https://doi.org/ 10.6061/clinics/2018/e210

84. Molina García FS, Zaragoza García E, Carrillo Badillo MP, Fernández de Santos AG, Calpena García A, Montoya Ventoso F. Implementación de la terapia láser endoscópica para las complicaciones de gestaciones gemelares monocoriales. Progresos en Obstet y Ginecol. 2009;52(6):313-319. https://doi.org/10.1016/ S0304-5013(09)71466-9

85. Morris RK, Selman TJ, Harbidge A, Martin WI, Kilby MD. Fetoscopic laser coagulation for severe twin-to-twin transfusion syndrome: factors influencing perinatal outcome, learning curve of the procedure and lessons for new centres. BJOG. 2010;117(11):1350-1357. https://doi.org/10.1111/j.1471-0528.2010.02680.x

86. Müllers SM, McAuliffe FM, Kent E, et al. Outcome following selective fetoscopic laser ablation for twin to twin transfusion syndrome: an 8 year national collaborative experience. Eur J Obstet Gynecol Reprod Biol. 2015;191:125-129. https://doi.org/10.1016/ j.ejogrb.2015.05.019

87. Nakata $M$, Ishii $K$, Sumie $M$, et al. A prospective pilot study of fetoscopic laser surgery for twin-to-twin transfusion syndrome between 26 and 27 weeks of gestation. Taiwan J Obstet Gynecol. 2016;55(4):512-514. https://doi.org/10.1016/j.tjog.2016.06.002

88. Nguyen HK, Cheng YW, Lee $\mathrm{H}$, et al. 446: recent experience with twin-twin transfusion syndrome: 2005-2011. Am J Obstet Gynecol. 2012;206(1):S205. https://doi.org/10.1016/j.ajog.2011.10.464

89. Ozawa K, Sugibayashi R, Wada S, et al. Fetoscopic laser photocoagulation for amniotic fluid discordance bordering on twin-twin transfusion syndrome: feasibility, perinatal and long-term outcomes. J Obstet Gynaecol Res. 2017;43(8):1256-1262. https://doi.org/10.1111/jog.13349

90. Papanna R, Johnson A, Ivey RT, Olutoye OO, Cass D, Moise KJ. Laparoscopy-assisted fetoscopy for laser surgery in twin-twin transfusion syndrome with anterior placentation. Ultrasound Obstet Gynecol. 2010;35(1):65-70. https://doi.org/10.1002/uog.7495

91. Papanna R, Habli M, Baschat AA, et al. Cerclage for cervical shortening at fetoscopic laser photocoagulation in twin-twin transfusion syndrome. Am J Obstet Gynecol. 2012;206(5):425. https://doi.org/10.1016/j.ajog.2012.02.022

92. Peeters SHP, Stolk TT, Slaghekke F, et al. latrogenic perforation of intertwin membrane after laser surgery for twin-to-twin transfusion syndrome. Ultrasound Obstet Gynecol. 2014;44(5):550-556. https://doi.org/10.1002/uog.13445

93. Persico N, Fabietti I, D'Ambrosi F, Riccardi M, Boito S, Fedele L. Postnatal survival after endoscopic equatorial laser for the treatment of twin-to-twin transfusion syndrome. Am J Obstet Gynecol. 2016;214(4):533. https://doi.org/10.1016/j.ajog.2015.10.020

94. Quintero RA, Comas C, Bornick PW, Allen MH, Kruger M. Selective versus non-selective laser photocoagulation of placental vessels in twin-to-twin transfusion syndrome. Ultrasound Obstet Gynecol. 2000;16(3):230-236. http://onlinelibrary.wiley.com/doi/10.1046/ j.1469-0705.2000.00265.x/full

95. Quintero RA, Bornick PW, Allen MH, Johson PK. Selective laser photocoagulation of communicating vessels in severe twin-twin transfusion syndrome in women with an anterior placenta. Obstet Gynecol. 2001;97(3):477-481.

96. Rossi AC, Kaufman MA, Bornick PW, Quintero RA. General vs local anesthesia for the percutaneous laser treatment of twin-twin transfusion syndrome. Am J Obstet Gynecol. 2008;199(2):137. https://doi.org/10.1016/j.ajog.2007.12.008

97. Ruano R, de Lourdes Brizot M, Liao AW, Zugaib M. Selective fetoscopic laser photocoagulation of superficial placental anastomoses for the treatment of severe twin-twin transfusion syndrome. Clinics (Sao Paulo). 2009;64(2):91-96. http://europepmc.org/search? query $=($ DOI:\%2210.1590/S1807-59322009000200005\%22)

98. Rüegg L, Hüsler M, Krähenmann F, Natalucci G, Zimmermann R, Ochsenbein-Kölble N. Outcome after fetoscopic laser coagulation in twin-twin transfusion syndrome-is the survival rate of at least one child at 6 months of age dependent on preoperative cervical length and preterm prelabour rupture of fetal membranes? J Matern Neonatal Med. 2018;0(0);1-9. https://doi.org/10.1080/14767058. 2018.1506441

99. Rustico MA, Lanna MM, Faiola S, et al. Fetal and maternal complications after selective fetoscopic laser surgery for twin-to-twin transfusion syndrome: a single-center experience. Fetal Diagn Ther. 2012;31(3):170-178. https://doi.org/10.1159/000336227

100. Said S, Flood K, Breathnach F, et al. Fetoscopic laser treatment of twin-to-twintransfusion syndrome (TTTS). Ir Med J. 2008;101(6): 191-193.

101. Senat M-V, Deprest J, Boulvain M, Paupe A, Winer N, Ville Y. Endoscopic laser surgery versus serial amnioreduction for severe twin-to-twin transfusion syndrome. N Engl J Med. 2004;351(2): 136-144.

102. Sepulveda W, Wong AE, Dezerega V, Devoto JC, Alcalde JL. Endoscopic laser surgery in severe second-trimester twin-twin transfusion syndrome: a three-year experience from a Latin American center. Prenat Diagn. 2007;27(11):1033-1038.

103. Shamshirsaz AA, Javadian P, Ruano R, et al. Comparison between laparoscopically assisted and standard fetoscopic laser ablation in patients with anterior and posterior placentation in twin-twin transfusion syndrome: a single center study. Prenat Diagn. 2015;35(4):376-381. https://doi.org/10.1002/pd.4552

104. Slaghekke F, Lopriore E, Lewi L, et al. Fetoscopic laser coagulation of the vascular equator versus selective coagulation for twin-to-twin 
transfusion syndrome: an open-label randomised controlled trial. Lancet. 2014;383(9935):2144-2151. https://doi.org/10.1016/ S0140-6736(13)62419-8

105. Taniguchi K, Sumie M, Sugibayashi R, Wada S, Matsuoka K, Sago H. Twin anemia-polycythemia sequence after laser surgery for twin-twin transfusion syndrome and maternal morbidity. Fetal Diagn Ther. 2015;37(2):148-153. https://doi.org/10.1159/000365812

106. Tchirikov M, Oshovskyy V, Steetskamp J, Falkert A, Huber G, Entezami M. Neonatal outcome using ultrathin fetoscope for laser coagulation in twin-to-twin-transfusion syndrome. J Perinat Med. 2011;39(6):725-730. https://doi.org/10.1515/JPM.2011.091

107. Teoh M, Walker S, Cole S, Edwards A. "A problem shared is a problem halved": success of a statewide collaborative approach to fetal therapy. Outcomes of fetoscopic laser photocoagulation for twin-twin transfusion syndrome in Victoria. Aust N Z J Obstet Gynaecol. 2013;53(2):108-113. https://doi.org/10.1111/ajo.12062

108. Thia E, Thain S, Yeo G. Fetoscopic laser photocoagulation in twin-to-twin transfusion syndrome: experience from a single institution. Singapore Med J. 2017;58(6):321-326. https://doi.org/ 10.11622/smedj.2016067

109. Ville Y, Van Peborgh P, Gagnon A, Frydman R, Fernandez H. Surgical treatment of twin-to-twin transfusion syndrome: coagulation of anastomoses with a Nd:YAG laser, under endosonographic control. Forty four cases. J Gynecol Obstet Biol Reprod. 1997;26(2):175-181.

110. Ville Y, Hecher K, Gagnon A, Sebire N, Hyett J, Nicolaides K. Endoscopic laser coagulation in the management of severe twin-to-twin transfusion syndrome. Br J Obstet Gynaecol. 1998;105(4): 446-453.

111. Weingertner A-S, Kohler A, Mager C, et al. Fetoscopic laser coagulation in 100 consecutive monochorionic pregnancies with severe twin-to-twin transfusion syndrome. J Gynecol Obstet Biol Reprod. 2011;40(5):444-451. https://doi.org/10.1016/j.jgyn.2011. 04.001

112. Wilson I, Henry A, Hinch E, et al. Audit of immediate outcomes for MCDA twins following laser therapy for twin-twin transfusion syndrome at the NSW fetal therapy centre. Aust N Z J Obstet Gynaecol. 2016;56(3):289-294. https://doi.org/10.1111/ajo.12464

113. Yamamoto M, El Murr L, Robyr R, Leleu F, Takahashi Y, Ville Y. Incidence and impact of perioperative complications in 175 fetoscopy-guided laser coagulations of chorionic plate anastomoses in fetofetal transfusion syndrome before 26 weeks of gestation. Am J Obstet Gynecol. 2005;193(3):1110-1116.

114. Yang X, Leung TY, Ngan Kee WD, Chen M, Chan LW, Lau TK. Fetoscopic laser photocoagulation in the management of twin-twin transfusion syndrome: local experience from Hong Kong. Hong Kong Med J. 2010;16(4):275-281.

115. Zaretsky MV, Tong SH, Lagueux M, et al. North American fetal therapy network: indications for delivery following laser ablation for twin-twin transfusion syndrome. Am J Obstet Gynecol. 2018;218(1): S316-S316. https://doi.org/10.1016/j.ajog.2017.11.055

116. Zhao D, Cohen D, Middeldorp JM, et al. Histologic chorioamnionitis and funisitis after laser surgery for twin-twin transfusion syndrome. Obstet Gynecol. 2016;128(2):304-312. https://doi.org/10.1097/ AOG.0000000000001469

117. Bebbington MW, Danzer E, Moldenhauer J, Khalek N, Johnson MP. Radiofrequency ablation vs bipolar umbilical cord coagulation in the management of complicated monochorionic pregnancies. Ultrasound Obstet Gynecol. 2012;40(3):319-324. https://doi.org/ 10.1002/uog.11122

118. Berg C, Holst D, Mallmann MR, Gottschalk I, Gembruch U, Geipel A. Early vs late intervention in twin reversed arterial perfusion sequence. Ultrasound Obstet Gynecol. 2014;43(1):60-64. https://doi. org/10.1002/uog.12578

119. Delabaere A, Favre N, Velemir L, et al. Suivi pédiatrique de 30 grossesses gémellaires monochoriales consécutives traitées par fœticide sélectif. Gynecol Obstet Fertil. 2013;41(2):85-89. https://doi.org/10.1016/j.gyobfe.2012.12.007

120. Deprest JA, Audibert F, Van Schoubroeck D, Hecher K, Mahieu-Caputo D. Bipolar coagulation of the umbilical cord in complicated monochorionic twin pregnancy. Am J Obstet Gynecol. 2000;182(2):340-345. https://doi.org/10.1016/S0002-9378(00) 70221-3

121. Gallot D, Laurichesse H, Lemery D. Selective feticide in monochorionic twin pregnancies by ultrasound-guided umbilical cord occlusion. Ultrasound Obstet Gynecol. 2003;22(5):484-488. https://doi.org/10.1002/uog.917

122. Gouverneur M, Klumper F, Loproire E, Vandenbussche F, Oepkes D. Selectieve foeticide door navelstrengcoagulatie bij afwijkende monochoriale meerlingen. Ned Tijdschr Geneeskd. 2009;153(19): 906-911.

123. Gül A, Güngördük K, Yildirim G, et al. Bipolar cord coagulation in monochorionic twins discordant for major fetal anomalies. J Turkish Ger Gynecol Assoc. 2008;9(1):24-28.

124. Has R, Kalelioglu I, Esmer AC, et al. Bipolar cord coagulation in the management of complicated monochorionic twin pregnancies. Fetal Diagn Ther. 2014;36(3):190-195. https://doi.org/10.1159/ 000360853

125. He ZM, Fang Q, Yang YZ, et al. Fetal reduction by bipolar cord coagulation in managing complicated monochorionic multiple pregnancies: preliminary experience in China. Chin Med J (Engl). 2010;123(5):549-554. https://doi.org/10.3760/cma.j.jissn.0366-6999. 2010.05.008

126. Ilagan JG, Wilson RD, Bebbington M, et al. Pregnancy outcomes following bipolar umbilical cord cauterization for selective termination in complicated monochorionic multiple gestations. Fetal Diagn Ther. 2008;23(2):153-158. https://doi.org/10.1159/ 000111598

127. Jelin E, Hirose S, Rand L, et al. Perinatal outcome of conservative management versus fetal intervention for twin reversed arterial perfusion sequence with a small acardiac twin. Fetal Diagn Ther. 2010;27(3):138-141. https://doi.org/10.1159/000295176

128. King JR, Conturie CL, Ouzounian JG, Korst LM, Llanes A, Chmait RH. Umbilical cord occlusion via laser coagulation in monochorionic multifetal gestations before and after 20 weeks of gestation. Fetal Diagn Ther. 2017;42(1):9-16. https://doi.org/10.1159/ 000448948

129. Lanna MM, Rustico MA, Dell'Avanzo M, et al. Bipolar cord coagulation for selective feticide in complicated monochorionic twin pregnancies: 118 consecutive cases at a single center. Ultrasound Obstet Gynecol. 2012;39(4):407-413. https://doi.org/10.1002/uog.11073

130. Lee H, Bebbington M, Crombleholme TM. The North American fetal therapy network registry data on outcomes of radiofrequency ablation for twin-reversed arterial perfusion sequence. Fetal Diagn Ther. 2013;33(4):224-229. https://doi.org/10.1159/000343223

131. Lewi L, Gratacos E, Ortibus E, et al. Pregnancy and infant outcome of 80 consecutive cord coagulations in complicated monochorionic multiple pregnancies. Am J Obstet Gynecol. 2006;194(3):782-789. http://www.hlisd.org/LibraryDetail.aspx?LibraryID=3866

132. Moise KJKY, Johnson A, Nickeleit V, et al. Radiofrequency ablation for selective reduction in the complicated monochorionic gestation. Am J Obstet Gynecol. 2008;198(2):198.e1-198.e5. https://doi.org/ 10.1016/j.ajog.2007.07.043 
133. Nobili E, Paramasivam G, Kumar S. Outcome following selective fetal reduction in monochorionic and dichorionic twin pregnancies discordant for structural, chromosomal and genetic disorders. Aust N Z J Obstet Gynaecol. 2013;53(2):114-118. https://doi.org/ 10.1111/ajo.12071

134. Paramasivam G, Wimalasundera R, Wiechec M, Zhang E, Saeed F, Kumar S. Radiofrequency ablation for selective reduction in complex monochorionic pregnancies. BJOG. 2010;117(10):1294-1298. https://doi.org/10.1111/j.1471-0528.2010.02624.x

135. Peng R, Xie HN, Lin MF, et al. Clinical outcomes after selective fetal reduction of complicated monochorionic twins with radiofrequency ablation and bipolar cord coagulation. Gynecol Obstet Invest. 2016;81(6):552-558. https://doi.org/10.1159/000445291

136. Quintero RA, Romero R, Reich $\mathrm{H}$, et al. In utero percutaneous umbilical cord ligation in the management of complicated monochorionic multiple gestations. Ultrasound Obstet Gynecol. 1996;8(1):16-22. http://onlinelibrary.wiley.com/doi/10.1046/ j.1469-0705.1996.08010016.x/full

137. Quintero RA, Chmait RH, Murakoshi T, et al. Surgical management of twin reversed arterial perfusion sequence. Am J Obstet Gynecol. 2006;194(4):982-991. https://doi.org/10.1016/j.ajog.2005.10.195

138. Roman A, Papanna R, Johnson A, et al. Selective reduction in complicated monochorionic pregnancies: radiofrequency ablation vs. bipolar cord coagulation. Ultrasound Obstet Gynecol. 2010;36(1):37-41. https://doi.org/10.1002/uog.7567

139. Schou KV, Jensen LN, Jørgensen C, Søgaard K, Tabor A, Sundberg K. Ultrasound-guided bipolar umbilical cord occlusion in complicated monochorionic pregnancies: is there a learning curve. Fetal Diagn Ther. 2018;44(1):65-71. https://doi.org/10.1159/000479104

140. Sugibayashi R, Ozawa K, Sumie M, Wada S, Ito Y, Sago H. Forty cases of twin reversed arterial perfusion sequence treated with radio frequency ablation using the multistep coagulation method: a single-center experience. Prenat Diagn. 2016;36(5):437-443. https://doi.org/10.1002/pd.4800

141. Takano M, Murata S, Fujiwara M, Hirata H, Nakata M. Experience of fetoscopic laser photocoagulation and cord transection for twin-reversed arterial perfusion sequence. J Obstet Gynaecol Res. 2015;41(9):1326-1329. https://doi.org/10.1111/jog.12720

142. Taylor MJO, Shalev E, Tanawattanacharoen S, et al. Ultrasound-guided umbilical cord occlusion using bipolar diathermy for stage III/IV twin-twin transfusion syndrome. Prenat Diagn. 2002;22(1):70-76.

143. Tsao KJ, Feldstein VA, Albanese CT, et al. Selective reduction of acardiac twin by radiofrequency ablation. Am J Obstet Gynecol. 2002;187(3):635-640. https://doi.org/10.1067/mob.2002.125242

144. Zhang ZT, Yang T, Liu CX, Li N. Treatment of twin reversed arterial perfusion sequence with radiofrequency ablation and expectant management: a single center study in China. Eur J Obstet Gynecol Reprod Biol. 2018;225:9-12. https://doi.org/10.1016/j.ejogrb. 2018.03.046

145. Deprest J, Jani J, Gratacos E, et al. Fetal intervention for congenital diaphragmatic hernia: the European experience. Semin Perinatol. 2005;29(2):94-103.

146. Harrison M, Keller R, Hawgood S. A randomized trial of fetal endoscopic tracheal occlusion for severe fetal congential diaphragmatic hernia. N Engl J Med. 2003;349(20):1916-1924.

147. Jani J, Gratacós E, Greenough A, et al. Percutaneous fetal endoscopic tracheal occlusion (FETO) for severe left-sided congenital diaphragmatic hernia. Clin Obstet Gynecol. 2005;48(4):910-922.

148. Jani JC, Nicolaides KH, Gratacós E, Vandecruys H, Deprest JA, Group FT. Fetal lung-to-head ratio in the prediction of survival in severe left-sided diaphragmatic hernia treated by fetal endoscopic tracheal occlusion (FETO). Am J Obstet Gynecol. 2006;195(6): 1646-1650.

149. Jani JC, Nicolaides KH, Gratacós E, et al. Severe diaphragmatic hernia treated by fetal endoscopic tracheal occlusion. Ultrasound Obstet Gynecol. 2009;34(3):304-310. https://doi.org/10.1002/uog.6450

150. Jiménez JA, Eixarch $E$, DeKoninck $P$, et al. Balloon removal after fetoscopic endoluminal tracheal occlusion for congenital diaphragmatic hernia. Am J Obstet Gynecol. 2017;217(1):78. https://doi.org/10.1016/j.ajog.2017.02.041

151. Kosinski P, Wielgos M. Foetoscopic endotracheal occlusion (FETO) for severe isolated left-sided congenital diaphragmatic hernia: single center polish experience. J Matern Neonatal Med. 2018;31(19):2521-2526. https://doi.org/10.1080/14767058.2017.1344969

152. Manrique S, Munar F, Andreu E, et al. Fetoscopic tracheal occlusion for the treatment of severe congenital diaphragmatic hernia: preliminary results. Rev Esp Anestesiol Reanim. 2008;55(7):407-413.

153. Peralta CFA, Sbragia L, Bennini JR, et al. Fetoscopic endotracheal occlusion for severe isolated diaphragmatic hernia: initial experience from a single clinic in Brazil. Fetal Diagn Ther. 2011;29(1):71-77. https://doi.org/10.1159/000314617

154. Persico N, Fabietti I, Ciralli F, et al. Fetoscopic Endoluminal tracheal occlusion in fetuses with severe diaphragmatic hernia: a three-year single-center experience. Fetal Diagn Ther. 2017;41(3):215-219. https://doi.org/10.1159/000448096

155. Ruano R, da Silva MM, Campos JADB, et al. Fetal pulmonary response after fetoscopic tracheal occlusion for severe isolated congenital diaphragmatic hernia. Obstet Gynecol. 2012;119(1):93-101. https://doi.org/10.1097/AOG.0b013e31823d3aea

156. Ruano R, Yoshisaki CT, da Silva MM, et al. A randomized controlled trial of fetal endoscopic tracheal occlusion versus postnatal management of severe isolated congenital diaphragmatic hernia. Ultrasound Obstet Gynecol. 2012;39(1):20-27. https://doi.org/ 10.1002/uog.10142

157. Ruano R, Peiro JL, da Silva MM, et al. Early fetoscopic tracheal occlusion for extremely severe pulmonary hypoplasia in isolated congenital diaphragmatic hernia: preliminary results. Ultrasound Obstet Gynecol. 2013;42(1):70-76. https://doi.org/10.1002/uog.12414

158. Arens C, Koch C, Veit M, et al. Anesthetic management for percutaneous minimally invasive fetoscopic surgery of spina bifida aperta: a retrospective, descriptive report of clinical experience. Anesth Analg. 2017;125(1):219-222.

159. Belfort MA, Whitehead WE, Shamshirsaz AA, et al. Fetoscopic open neural tube defect repair: development and refinement of a two-port, carbon dioxide insufflation technique. Obstet Gynecol. 2017;129(4):734-743.

160. Degenhardt J, Schürg R, Winarno $A$, et al. Percutaneous minimal-access fetoscopic surgery for spina bifida aperta. Part II: maternal management and outcome. Ultrasound Obstet Gynecol. 2014;44(5):525-531. https://doi.org/10.1002/uog.13389

161. Kohn J, Rao V, Sellner A, et al. Management of labor and delivery after fetoscopic repair of an open neural tube defect. Obstet Gynecol. 2018;131(6):1062-1068.

162. Pedreira DAL, Zanon N, de Sá RAM, et al. Fetoscopic single-layer repair of open spina bifida using a cellulose patch: preliminary clinical experience. J Matern Fetal Neonatal Med. 2014;27(16):1613-1619. https://doi.org/10.3109/14767058.2013.871701

163. Pedreira DAL, Zanon N, Nishikuni K, et al. Endoscopic surgery for the antenatal treatment of myelomeningocele: the CECAM trial. Am J Obstet Gynecol. 2016;214(1):111. https://doi.org/10.1016/ j.ajog.2015.09.065 
164. Verbeek RJ, Heep A, Maurits NM, et al. Fetal endoscopic myelomeningocele closure preserves segmental neurological function. Dev Med Child Neurol. 2012;54(1):15-22. https://doi.org/ 10.1111/j.1469-8749.2011.04148.x

165. Ziemann M, Fimmers R, Khaleeva A. Partial amniotic carbon dioxide insufflation $(\mathrm{PACl})$ during minimally invasive fetoscopic interventions on fetuses with spina bifida aperta. Surg Endosc. 2018;32(7):3138-3148. Epub ahead. https://doi.org/10.1007/s00464018-6029-z

166. Morris RK, Malin GL, Quinlan-Jones E, et al. Percutaneous vesicoamniotic shunting versus conservative management for fetal lower urinary tract obstruction (PLUTO): a randomised trial. Lancet. 2013;382(9903):1496-1506. https://doi.org/10.1016/ S0140-6736(13)60992-7

167. Ruano R, Duarte S, Bunduki V, Giron AM, Srougi M, Zugaib M. Fetal cystoscopy for severe lower urinary tract obstruction--initial experience of a single center. Prenat Diagn. 2010;30(1):30-39. https://doi.org/10.1002/pd.2418

168. Welsh A, Agarwal S, Kumar S, Smith RP, Fisk NM. Fetal cystoscopy in the management of fetal obstructive uropathy: experience in a single European Centre. Prenat Diagn. 2003;23(13):1033-1041. https://doi.org/10.1002/pd.717

169. Cavalheiro S, Moron AF, Almodin CG, et al. Fetal hydrocephalus. Childs Nerv Syst. 2011;27(10):1575-1583. https://doi.org/10.1007/ s00381-011-1539-1

170. Mallmann MR, Graham V, Rösing B, et al. Thoracoamniotic shunting for fetal hydrothorax: predictors of intrauterine course and postnatal outcome. Fetal Diagn Ther. 2017;41(1):58-65. https://doi.org/ 10.1159/000446110

171. Kohl T, Hering R, Van de Vondel P, et al. Analysis of the stepwise clinical introduction of experimental percutaneous fetoscopic surgical techniques for upcoming minimally invasive fetal cardiac interventions. Surg Endosc. 2006;20(7):1134-1143.

172. Kohl T, Tchatcheva K, Weinbach J, et al. Partial amniotic carbon dioxide insufflation (PACl) during minimally invasive fetoscopic surgery: early clinical experience in humans. Surg Endosc. 2010;24(2):432-444. https://doi.org/10.1007/s00464-009-0579-z

173. Nivatpumin P, Pangthipampai $P$, Jirativanont T, Dej-Arkom $S$, Triyasunant N, Tempeetikul T. Anesthetic techniques and incidence of complications in fetoscopic surgery. I Med Assoc Thai. 2016;99(5):602-610.

174. Peralta CFA, Sbragia L, Corrêa-Silva EP, et al. Maternal complications following endoscopic surgeries in fetal medicine. Rev Bras Ginecol Obstet. 2010;32(6):260-266.

175. Thom EA. Maternal reproductive outcomes after in-utero repair of myelomeningocele. Am J Obstet Gynecol. 2016;214(1).

176. Wilson RD, Lemerand K, Johnson MP, et al. Reproductive outcomes in subsequent pregnancies after a pregnancy complicated by open maternal-fetal surgery (1996-2007). Am J Obstet Gynecol. 2010;203(3):209. https://doi.org/10.1016/j.ajog.2010.03.029

177. Gregoir C, Engels AC, Gomez O, et al. Fertility, pregnancy and gynecological outcomes after fetoscopic surgery for congenital diaphragmatic hernia. Hum Reprod. 2016;31(9):2024-2030. https://doi.org/10.1093/humrep/dew160

178. Le Lous M, Mediouni I, Chalouhi G, et al. Impact of laser therapy for twin-to-twin transfusion syndrome on subsequent pregnancy. Prenat Diagn. 2018;38(4):293-297. https://doi.org/10.1002/pd.5227
179. Vergote S, Lewi L, Gheysen W, De Catte L, Devlieger R, Deprest J. Subsequent fertility, pregnancy, and gynecologic outcomes after fetoscopic laser therapy for twin-twin transfusion syndrome compared with normal monochorionic twin gestations. Am J Obstet Gynecol. 2018;218(4):447.e1-447.e7. https://doi.org/10.1016/j.ajog.2018.01.013

180. Mascarenhas MN, Flaxman SR, Boerma T, Vanderpoel S, Stevens GA. National, regional, and global trends in infertility prevalence since 1990: a systematic analysis of 277 health surveys. PLoS Med. 2012;9(12):1-12. https://doi.org/10.1371/journal.pmed.1001356

181. Wilcox A, Weinber C, O'Connor J, Baird D. Incidence of early pregnancy loss. N Engl J Med. 1988;319(4):189-194.

182. Andersen AN, Wohlfahrt J, Christens P, Olsen J, Melbye M, Nybo A. Maternal age and fetal loss: population based register linkage study. BMJ. 2000;320(June):1708-1712.

183. Herbert D, Lucke J, Dobson A. Pregnancy losses in young Australian women. Findings from the Australian longitudinal study on Women's health. Womens Health Issues. 2009;19(1):21-29. https://doi.org/ 10.1016/j.whi.2008.08.007

184. Blencowe H, Cousens S, Oestergaard MZ, et al. National, regional, and worldwide estimates of preterm birth rates in the year 2010 with time trends since 1990 for selected countries: a systematic analysis and implications. Lancet. 2012;379(9832):2162-2172. https://doi.org/ 10.1016/S0140-6736(12)60820-4

185. Tanne JH. Preterm and low weight births rise again in the US. BMJ. 2017;3311:j3311. https://doi.org/10.1136/bmj.j3311

186. National Institute for Health and Care Excellence. Preterm labour and birth. 2015. nice.org.uk/guidance/ng25.

187. Greene RA, Fitzpatrick C, Turner MJ. What are the maternal implications of a classical caesarean section? J Obstet Gynaecol. 1998;18(4):345-347. https://doi.org/10.1080/01443619867083

188. Shavit C, Rollins M, Ferschl M. Maternal convulsion during high-dose sevoflurane anaesthesia for open foetal surgery. $\mathrm{Br} J$ Anaesth. 2017;118(4):634-635.

189. Gratacós E, Deprest J. Current experience with fetoscopy and the Eurofoetus registry for fetoscopic procedures. Eur J Obstet Gynecol Reprod Biol. 2000;92(1):151-159.

190. NAFTNet Website. https://www.naftnet.org.

191. Bliton MJ. Parental hope confronting scientific uncertainty: a test of ethics in maternal-fetal surgery for spina bifida. Clin Obstet Gynecol. 2005;48(3):595-607.

192. Fry JT, Frader JE. "We want to do everything": how parents represent their experiences with maternal-fetal surgery online. J Perinatol. 2018;38(3):226-232. https://doi.org/10.1038/s41372-017-0040-4

\section{SUPPORTING INFORMATION}

Additional supporting information may be found online in the Supporting Information section at the end of the article.

How to cite this article: Sacco A, Van der Veeken L, Bagshaw $E$, et al. Maternal complications following open and fetoscopic fetal surgery: A systematic review and meta-analysis. Prenatal Diagnosis. 2019;39:251-268. https://doi.org/10.1002/pd.5421 